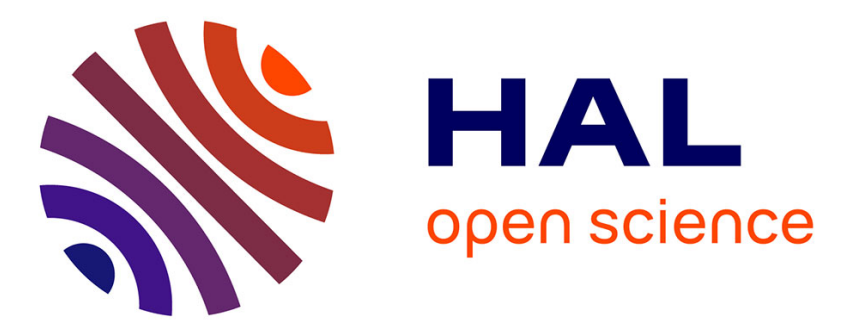

\title{
Optimal Ecodriving Control: Energy-Efficient Driving of Road Vehicles as an Optimal Control Problem
}

\author{
A Sciarretta, Giovanni de Nunzio, L Leon Ojeda
}

\section{To cite this version:}

A Sciarretta, Giovanni de Nunzio, L Leon Ojeda. Optimal Ecodriving Control: Energy-Efficient Driving of Road Vehicles as an Optimal Control Problem. IEEE Control Systems Magazine, 2015, 35

(5), pp.71-90. 10.1109/MCS.2015.2449688 . hal-01252197

\section{HAL Id: hal-01252197 \\ https://hal-ifp.archives-ouvertes.fr/hal-01252197}

Submitted on 7 Jan 2016

HAL is a multi-disciplinary open access archive for the deposit and dissemination of scientific research documents, whether they are published or not. The documents may come from teaching and research institutions in France or abroad, or from public or private research centers.
L'archive ouverte pluridisciplinaire HAL, est destinée au dépôt et à la diffusion de documents scientifiques de niveau recherche, publiés ou non, émanant des établissements d'enseignement et de recherche français ou étrangers, des laboratoires publics ou privés. 


\title{
Optimal Eco-Driving Control \\ Energy-Efficient Driving of Road Vehicles as an Optimal Control Problem
}

\author{
A. Sciarretta, G. De Nunzio, and L. Leon Ojeda
}

June 10, 2015

Transportation is responsible for a substantial fraction of worldwide energy consumption and greenhouse gas emissions. It is the largest sector after energy production. However, while emissions from other sectors are generally decreasing, those from transportation have increased since 1990. Reducing the impact of transportation is a task that is inherently associated with the improvement of energy efficiency, particularly for passenger cars that contribute to almost half of the whole sector.

At least three energy conversion steps are relevant for a comprehensive analysis of energy efficiency of passenger cars. As illustrated in Figure 1, in a first step ("grid-to-tank"), energy carriers that are available at stationary distribution nodes are converted to an energy carrier that is suitable for onboard storage, such as gasoline or electricity. This energy is then converted by the propulsion system to mechanical energy aimed at propelling the vehicle ("tank-to-wheels"). In the third energy conversion step ("wheelto-meters"), this mechanical energy is ultimately converted into the kinetic and potential energy required by the displacement. Unfortunately, all of these conversion processes cause substantial energy losses.

Tank-to-wheels efficiency may be improved by several approaches, both at the component level and at the system control level [1]. Methods to improve grid-to-tank efficiency by choosing the appropriate charging slots and profiles are currently being studied for electric vehicles [2]. This article focuses only on wheel-to-meters efficiency, which is influenced by vehicle parameters (such as weight, aerodynamic drag, rolling friction) and the driving profiles followed by the vehicle. As Table 1 shows, the latter play an important role in determining energy losses. The approach of improving wheel-to-meters efficiency by "controlling" the driving profile reveals its potential when considering that it does not require structural changes to the system. 
The adoption of an energy-aware driving style is the goal of "eco-driving" training courses. These courses are typically based on a few heuristic guidelines, for instance, anticipate traffic flow, avoid braking, shift up early, switch off the engine at short stops [3]. In addition, software tools and systems that help the driver (or replace the driver) in performing eco-driving have also emerged. A possible classification of existing eco-driving systems is [4]:

1. Pre-trip systems, besides giving generic advice on eco-driving, are integrated within navigation systems guiding the driver on the most energy-efficient route.

2. In-trip systems are part of the broader category of advanced driverassistance systems (ADAS) and are further classified as (2.a) online assessment systems, which provide feedback advice based on current performance, (2.b) online advice systems, which give a predictive, feedforward advice, based on upcoming events, and (2.c) predictive cruise controllers, where automatic driving is performed. As a main interface, the majority of systems (2.a) and (2.b) use visual displays while audible and haptic gas pedal are applied in only a few solutions.

3. After the trip, post-trip systems attempt to increase the driver's motivation for eco-driving by displaying encouraging results and generating summaries and statistics that can be compared to other drivers.

Most current approaches are based on heuristic rules of thumb or good practices that are associated with an energy-efficient drive [5]. Moreover, only a few are predictive, that is, based on estimations of future external conditions, while the rest are solely based on current driving information, typically extracted from vehicle's network data. Even more rudimentary concepts, essentially consisting of alerts based on the acceleration sensor of smartphones, are typically found among mobile application software labelled "eco-drive."

However, several concepts are emerging that attempt at implementing eco-driving in a more rigorous framework, and are the subject of this article. In these concepts, eco-driving is regarded as an optimal control problem where the drive commands minimize the energy consumption for a given trip.

In this article, a general formulation of an optimal control problem is presented that covers, with little or no adjustements, several distinct scenarios. To start with, powertrain modeling is reviewed, then the optimal control problem formulation is presented. Some solution techniques are described and typical optimization results are presented. The article finally 
discusses some experimental implementations of the ideas presented. While the article is generally aimed at reviewing and reorganizing published material, some original contributions are indeed presented, namely, an analytical solution of the optimal eco-driving problem for hybrid-electric vehicles and a parametric optimization method for conventional vehicles.

\section{Context and Scenarios}

Although the practical application of optimal control theory to the driving profiles of passenger cars is quite recent, it is interesting that in many respects this problem is similar to the century-old Goddard problem of minimizing the fuel consumption of a rocket in vertical flight for a given summit altitude [6]. This problem and its numerous variants have been long studied [7-9] and still are, since they have become a classical benchmark example in optimal control due to the characteristic control behavior in connection with a relatively simple model structure [10]. In fact, early works already had shown that the optimal flight profiles consist of three thrust regimes. Singular regimes, for which the thrust is neither zero nor equal to its maximum value, were found.

In addition, optimal running profiles for off-road vehicles [1] or road vehicles running on prescribed routes, such as trains [12], busses [13] or race cars [14,15] have been extensively studied as well. In such scenarios, the driving conditions are reasonably well known in advance, and optimal speed profiles can be calculated offline with an acceptable confidence and possibly implemented in an open-loop or state-feedback controller. In contrast, passenger cars and other vehicles on open roads experience a wide range of driving conditions, most of which are unknown in advance. Likely for such reasons, the application of energy-optimal drive control to such scenarios has been thoroughly investigated only in the last two decades [16].

Several studies [17 22 focus on applications on highways, with high average speeds and varying road slopes. The trip destination is known from a navigation system, together with route information including altitude and vehicle positioning. The speed profile is regulated to anticipate altitude variations, however traffic and vehicle stop or starts only play a small role in this approach.

An emerging litterature [3,23, 28, treats urban driving, where the vehicle trajectory is constrained by the infrastructure (road signs and signals) and other vehicles (traffic). These constraints are predictable to some extent through the use of appropriate sensors, and have a major impact on the 
calculation of the optimal vehicle trajectories.

Urban conditions are also characterized by infrastructure constraints that are variable with time, as it is the case of traffic lights. Such circumstance has inspired works aiming at optimizing the approach to a traffic light that transmits information on its status [29], or the "green-waving" between sequences of communicating traffic lights [30 35]. Besides energy economy of each single vehicle, fluidification of traffic can be regarded as an indirect optimization objective in such scenario.

Yet a different scenario is the energy-efficient cruise control, where the vehicle has to be driven in the most efficient way while keeping its speed within a certain band that is variable according to the leading vehicle's behavior [36 38]. Additionally, safety concerns impose inter-vehicle distance constraints.

\section{Powertrains and Their Modeling}

The concept of drive control applies to internal-combustion-engine-powered vehicles (ICEV) and particularly to ICE-powered trucks, electric vehicles (EV), and hybrid-electric vehicles (HEV). Other types of vehicles, such as hydraulic-hybrid vehicles, are promising for energy-optimal drive control due to their typical application fields (for instance, delivery vehicles) [39].

For all types of powertrain, the energy demand at the wheels is

$$
E_{\mathrm{dem}}=\int_{0}^{t} v(t) F_{w}(t) d t
$$

where $v$ is the vehicle's speed and $F_{w}$ is the traction force transmitted by the powertrain to the wheels. The vehicle's longitudinal dynamics are

$$
m \frac{d v(t)}{d t}=F_{w}(t)-F_{\text {res }}(t)-F_{b}(t)
$$

where $m$ includes the effect of the inertia of the rotating parts (wheels, engine, motor), which is possibly varying with time, and $F_{b}$ is the mechanical brake force. The resistance force is calculated as

$$
F_{\text {res }}(t)=\frac{1}{2} \rho_{a} c_{d} A_{f} v(t)^{2}+c_{r} m g+m g \sin (\alpha(s(t))),
$$

where $\rho_{a}$ is the air density, $c_{d}$ is the aerodynamic drag coefficient, $A_{f}$ is the vehicle's frontal area, $c_{r}$ is the rolling resistance coefficient, $g$ is the gravity acceleration, $\alpha$ is the road slope angle, and $s$ is the vehicle's position along 
the route. Alternatively, $F_{\text {res }}$ can be represented as a polynomial function of $v(t)$.

In a model covering at least ICEVs, EVs, and parallel HEVs (see "Vehicle Powertrain Architectures" for a classification of HEV configurations), the powertrain force results from the combination of the engine torque and the motor torque,

$$
F_{w}(t)=\frac{T_{n} \eta_{t}^{\operatorname{sign}\left(T_{n}(t)\right)}}{r_{w}}, \quad T_{n}:=\left(T_{m}(t) \gamma_{m}+T_{e}(t) \gamma_{e}(t)\right),
$$

where $T_{e}$ is the engine torque, $T_{m}$ is the motor torque, $r_{w}$ is the wheel radius, $\gamma_{e}$ and $\gamma_{m}$ are the transmission ratio of the engine and the motor, respectively, and $\eta_{t}$ is the transmission efficiency (that depends on the gear ratio used, although this dependency is often neglected). Models of ICEVs and EVs can be considered as particular cases of (4) with $T_{m}(t) \equiv 0$ and $T_{e}(t) \equiv 0$, respectively.

Assuming that a discrete transmission (gearbox) is used, $\gamma_{e}$ includes both the gearbox and the final drive, and varies with the gear selected either by the driver (manual transmissions) or by the transmission controller (automatic transmissions). In the latter case, the gear ratio is the result of a gear shift law that can be expressed as a map

$$
\gamma_{e}(t)=\Gamma\left(v(t), F_{w}(t)\right) .
$$

In the rest of the article, a fixed transmission ratio $\gamma_{m}$ is considered, representing simple reductor gears of EVs. Note that (4) implies a discontinuous derivative of $F_{w}$ with respect to $T_{n}$ at $T_{n}=0$ (coasting operation), which separates a traction operation $\left(T_{n}>0\right)$ from a braking operation $\left(T_{n}<0\right)$.

Engine and motor speeds, $\omega_{e}$ and $\omega_{m}$, are related to the vehicle's speed through their respective transmission ratios

$$
\omega_{e}(t)=\frac{\gamma_{e}(t)}{r_{w}} v(t) \quad \text { and } \quad \omega_{m}(t)=\frac{\gamma_{m}}{r_{w}} v(t) .
$$

In contrast with $E_{\mathrm{dem}}$, the consumption of the onboard stored energy, $E_{\text {tank }}$, depends on the nature of the powertrain.

\section{Internal Combustion Engine Vehicles}

In ICEVs, $E_{\text {tank }}=\int_{0}^{t} P_{f} d t$, where $P_{f}$ is the fuel power consumed by the engine. This quantity can be modeled under a steady-state approximation 
using tabulated data ("engine map") as a function of engine torque and rotational speed

$$
P_{f}(t)=P_{f}\left(T_{e}(t), \omega_{e}(t)\right) .
$$

When $v=0$ (vehicle stopped) or $\gamma_{e}=0$ (neutral gear engaged), the fuel consumption at idle must be considered [18]. For online implementation, approximate closed-form expressions are used instead of (7), for instance, polynomial expressions $[3,18,19,34,40]$ or the affine-in-torque Willans model [20, 34

$$
P_{f}(t)=\frac{T_{e}(t) \omega_{e}(t)+P_{f, 0}\left(\omega_{e}(t)\right)}{e\left(\omega_{e}(t)\right)}
$$

defined by the speed-dependent parameters $e$ (indicated efficiency, that is, the efficiency of the combustion process alone) and $P_{f, 0}$ (idle losses).

\section{Electric Vehicles}

In EVs, $E_{\text {tank }}=\int_{0}^{t} P_{b} d t$, where $P_{b}$ is the electrochemical power drained from or supplied to the battery.

Electric power supplied to or generated by the motor, including its control electronics, $P_{m}$, is usually tabulated ("motor map") as a function of motor torque and rotational speed

$$
P_{m}(t)=P_{m}\left(T_{m}(t), \omega_{m}(t)\right) .
$$

For online implementation, approximate closed-form expressions are used instead of (9). For instance, dc motor equations inspire the model [24, 25, 28, 41]

$$
P_{m}(t)=b_{2} T_{m}(t)^{2}+b_{1} T_{m}(t) v(t)+b_{1}^{\prime} T_{m}(t)+b_{0} v(t)+b_{0}^{\prime},
$$

where the $b_{i}$ 's are tunable parameters. Even simpler representations, for instance constant motoring and generating efficiency, have also been adopted in eco-driving studies [20,32].

The battery power is calculated by modeling the battery electrochemistry as an equivalent electric circuit, with an ideal voltage source $U_{b 0}$ and an internal resistance $R_{b}$ in series,

$$
P_{b}(t)=\frac{U_{b 0}^{2}}{2 R_{b}}-U_{b 0} \sqrt{\frac{U_{b 0}^{2}-4 P_{m}(t) R_{b}}{4 R_{b}^{2}}} .
$$

The difference between $P_{b}$ and $P_{m}$ is due to inner battery losses; in ecodriving studies these effects are sometimes further simplified to a constant efficiency model or neglected [20,32, 41]. 


\section{Hybrid-Electric Vehicles}

To model a HEV, models of the engine, the electric machine, and the battery presented in the previous sections still apply. These models lead to the calculation of fuel power $P_{f}$ and battery electrochemical power $P_{b}$, and the resulting energy consumption.

\section{Route and Traffic Information}

An insight into the model equations of the previous section reveals that the knowledge of the road slope $\alpha$ plays a key role in energy consumption minimization. The road slope is usually provided as a function of the vehicle location by some sort of geographic information system (GIS). The vehicle positioning is retrieved or assumed to be retrieved by global navigation satellite systems. More accurate data can be provided by online estimation of road slope of frequently traveled roads [42].

A similar key role is played by the knowledge of the vehicle mass. While mass is typically constant or slightly variable when considering passenger cars, for some applications such as buses, garbage trucks, or delivery trucks this parameter may be time dependent and thus has to be estimated. The literature presents many online vehicle mass estimators [43].

Establishment of constraints to the optimization problem requires additional pieces of information about the route. One example is top speed limits, which are either available from GIS or detected through onboard cameras and processing algorithms. Similarly, knowledge of road curvature can enforce speed limitations due to maximal centrifugal acceleration [20,26].

A different class of constraints is imposed by traffic, that is, by the leading vehicle on the route. The traffic-dictated speed limit is not just a function of the distance along the route but essentially a poorly predictable disturbance. Average speed of traffic flow ahead might be provided by realtime traffic reporting or traffic congestion maps. The specific knowledge of the leading vehicle speed requires the use of dedicated sensors such as the radar sensors used for adaptive cruise control (ACC).

Another constraint to vehicle trajectories may be imposed by traffic lights, depending on the scenario considered. The information on trafficlight state can be provided by infrastructure-to-vehicle (I2V) communication, that is, dedicated short-range communication (DSRC) technologies $[29,30,33$. The bidirectional scenario, that is, with vehicle-to-infrastructure (V2I) facilities, has been studied as well [44].

Independent studies [27] have shown that there is a correlation between 
stochastic events such as traffic or traffic lights, the route segment, the average speed, and the time during the day. Therefore, statistical tools ("energetic maps") can be adopted as an alternative to ACC, V2I, or I2V communication to forecast those events in an online context [28].

\section{Optimal Control Problem Formulation}

Energy-optimal drive control under several scenarios (acceleration, deceleration, driving between stops, approaching traffic lights, cruise within a speed band) [3] can be treated in a similar way by defining an eco-driving optimal control problem (ED-OCP).

\section{Optimization Horizon}

The main objective of the ED-OCP is to minimize fuel or energy consumption over a certain horizon. This horizon can be expressed in terms of time or distance. In highway, green-waving, and cruise control scenarios, time horizon $t_{f}$ is typically related to the telemetric preview length [19, 23, 31, 36, 38]. In other cases, the time horizon can be obtained from the distance horizon or other trip information by means of average speed, either desired by the driver or imposed by the traffic [20,41]. In simple traffic-light scenarios, $t_{f}$ might be the time to the next green period [29].

The spatial horizon $s_{f}$ may represent the overall trip length, particularly in highway-oriented applications [18, 20,22, 26], as well as in green-waving scenarios, but only if single intersections are marked by interior point constraints [33,34] (see below). In traffic-light scenarios, $s_{f}$ is the distance to the intersection [3, 29, 30, 35]. For EVs, $s_{f}$ can be set as the estimated distance to the next recharge station [27.

Both final time and distance are usually assigned to given horizons. Otherwise, when final time is prescribed, final distance can be free [31,36, 38] or maximized [19,41]. Conversely, when the final distance is to be prescribed, final time can be free [21, 22, 26, 34], constrained in a time window [30], or minimized $[17,18,27,33$, depending on the scenario considered. In any cases, final speed can be either free or prescribed.

\section{Objective Function}

Given the energy-oriented nature of the drive controller, a natural choice for the objective function of the ED-OCP is the energy consumption over the horizon chosen, where the energy consumption rate $P_{\text {tank }}$ coincides with $P_{f}$ 
(the fuel power) for ICEVs and HEVs, while $P_{\text {tank }}=P_{b}$ (the battery power) for EVs. Simpler formulations take the power at the wheels, or even purely kinematic terms [30,31,33] as the running cost. In addition, trip time may be minimized or trip distance maximized, as discussed below.

For highway scenarios, additional terms are sometimes appended to the objective function to penalize deviations from a reference average speed [17, 21], vehicle acceleration [18, or gear shifts [19]. Drivability and aging can be also appended to the objective functions [21,26].

\section{Control}

The argument of minimization are the control laws applied to drive the vehicle. For all types of powertrains, at least two control inputs exist: $F_{w}$ and $F_{b}$. Being mutually exclusive, these control inputs may be arranged in a single variable. Equivalent to wheel force are other variables sometimes chosen as control inputs, such as acceleration, fuel quantity, or motor voltage.

An alternative formulation is to set a desired or target speed as a control input [17, 27], where it is assumed that the driver regulates the pedals to achieve the target speed.

For ICEVs and HEVs with automatic gearboxes, also $\gamma_{e}$ might be present as a control input $[3,18,21,26,45]$. Clutch control is another possible discrete control [3] but it can conveniently be lumped either with the gear control input or with the engine control input 21.

Additional degrees of freedoms exist in HEVs. For instance, in parallel HEVs, an additional control input represents either the engine torque (in addition to wheel torque or force) [21,45] or, equivalently, the torque split ratio between the engine and the motor [23].

\section{State Dynamics}

In most formulations, a minimal set of system state variables includes vehicle speed and position. The former dynamics is given by (2)

$$
\dot{v}(t)=\frac{F_{w}(t)}{m}-\frac{1}{2 m} \rho_{a} c_{d} A_{f} v(t)^{2}-c_{r} g-g \sin (\alpha(s(t)))-\frac{F_{b}(t)}{m}, \quad v(0)=v_{i},
$$

while the spatial dynamics are

$$
\dot{s}(t)=v(t), \quad s(0)=0 .
$$


Equation (13) allows transforming a time-based ED-OCP into a positionbased ED-OCP via the transformation

$$
\frac{d}{d t}=\frac{1}{v} \frac{d}{d s}
$$

Depending on the degree of refinement of the model used, in some approaches additional dynamics are considered, such as engine speed [17] or gear ratio $18,21,26$.

For HEVs, an intrinsic additional state is the energy remaining in the battery, $\xi$. Its state dynamics are

$$
\dot{\xi}(t)=P_{b}(t), \quad \xi(0)=\xi_{i} .
$$

Equivalent formulations are in terms of battery state of charge (SoC), which is related to the battery energy by constant or weakly-varying parameters [1]. In some formulations for $\mathrm{EVs}, \mathrm{SoC}$ is a state variable and it is constrained at the end of the trip [27.

\section{Control Constraints}

Control inputs $T_{e}$ and $T_{m}$ are bounded by limits $T_{\{e, m\}, \max }(v(t))$ and $T_{\{e, m\}, \min }(v(t))$ that are typically varying with the vehicle speed, since both IC engines and electric motors have torque $\max / \mathrm{min}$ curves that are functions of their respective rotational speeds. In addition to these limits, traffic-constrained force or torque may be considered [23]. Force or acceleration rate can be also bounded [36].

The brake control input $F_{b}$ defined here as a positive quantity, see (2), is bounded between 0 and the maximal brake effort $F_{b, \max }$.

When the gear is selected by the drive controller, $\gamma_{\max }(t)$ and $\gamma_{\min }(t)$ are actually functions of the gear engaged, thus explaining why in some approaches [18] the gear itself is a state variable.

\section{State Constraints}

State variables are subject to inequality constraints. For the vehicle speed,

$$
v_{\min }(t, s(t)) \leq v(t) \leq v_{\max }(t, s(t)),
$$

where the quantity $v_{\max }$ is the most restrictive of several possible contributions, including top speed limit and subjective maximal speed allowed. A variable $v_{\max }(t, s(t))$ can be also used to describe traffic lights, by setting it 
to zero when the signal is red [32]. The variable quantity $v_{\min }$ is particularly relevant for highway scenarios [18, 19,41,45] and for cruise scenarios [3,33,38].

A different class of state constraints concerns the position. In multivehicle scenarios, $s(t)$ may be constrained by the inter-vehicle distance with a leading vehicle, characterized by multiple parameters [31, 37, 38]. Alternatively, cumulative inter-vehicle distance may be transferred to the objective function [36.

When relevant, the battery energy $\xi(t)$ or SoC is also limited in an admissible window depending on the battery technology.

\section{Terminal Constraints}

Terminal constraints concern the final values of time, position, and speed. For the former two, see section "Objective Function." Terminal speed is typically free in some scenarios such as highway driving [17-19, 41], cruise [3,36, 38], green-waving between traffic lights [29 31,33], or race circuits [14]. In other situations, it is constrained, more or less "rigidly," to be around a prescribed value $v_{f}$.

When a HEV is considered, an additional constraint concerns the final $\mathrm{SoC}$ or battery energy. The latter is prescribed to match the initial value in charge-sustaining hybrids, or a target minimal value in plug-in hybrids. However, when multiple segments are considered (urban-type scenarios), this constraint applies only at the end of the last segment.

Hard constraints can be replaced by "soft" constraints that are adjoint to the cost function as terminal penalty terms. Final position, final speed, and $\mathrm{SoC}$ can be penalized in this way $[21,27,32]$.

\section{Interior Constraints}

Equality (pointwise) constraints on both speed and position are typical of green-waving and urban displacement scenarios [34]. These constraints can be applied directly, for instance, $s\left(t_{b, j}\right)=s_{b, j}$, or enforced by appropriately setting the state boundaries described in section "State Constraints," for instance, $v_{\min }\left(t_{b, j}, s_{b, j}\right)=v_{\max }\left(t_{b, j}, s_{b, j}\right)=v_{b, j}$, where $t_{b, j}$ and $s_{b, j}, j=$ $1, \ldots, n_{b}$ are the time instants and positions at which the speed is imposed (by traffic lights or other events).

In these cases, an alternative approach to interior constraints consists of separating the trip into $n_{b}$ correlated segments for which $n_{b}$ correlated OCPs apply, each of which is characterized by its own temporal and spatial 
horizon, as well as by its own initial and terminal conditions

$$
t_{f, j}=t_{b, j}-t_{b, j-1}, \quad s_{f, j}=s_{b, j}-s_{b, j-1}, \quad v_{f, j}=v_{i, j+1}=v_{b, j} .
$$

with $t_{b, 0}=s_{b, 0}=0, v_{b, 0}=v_{i}, v_{b, n_{b}}=v_{f}$. The energy consumption of the whole trip is the sum of the energy consumption of each segment.

Segments are identified either by (i) changes in road characteristics, such as top speed limits or slope, or by (ii) driver- or traffic-induced events, such as planned stops, intersections, traffic lights, or traffic queues. All these segment identifiers are called breakpoints in the rest of this article [24,25,28, see Figure 2

\section{ED-OCP Formulation Summary}

With the considerations above, a general formulation of ED-OCP separated into $n_{b}$ subproblems for each single segment can be expressed as follows (the segment-counting subscript $j$ is omitted for simplicity). Find $u(t):=$ $\left\{T_{e}(t), T_{m}(t), F_{b}(t)\right\}$ such that

$$
\min _{u \in U(x(t))} J=\int_{0}^{t_{f}} g(u, x(\tau)) \mathrm{d} \tau
$$

with $x:=\{s, v, \xi\}, g(\cdot)=P_{\text {tank }}$ given by (8) or (10)-(11), subject to

$$
\dot{x}(t)=f(u(t), x(t))
$$

with $f(\cdot)$ given by (12)-(13),

$$
\begin{gathered}
v_{\min }(t, s(t)) \leq v(t) \leq v_{\max }(t, s(t)), \quad 0<s(t)<s_{f}, \quad \xi_{\min }<\xi(t)<\xi_{\max }, \\
v(0)=v_{i}, \quad s(0)=0
\end{gathered}
$$

and

$$
v\left(t_{f}\right)=v_{f}, \quad s\left(t_{f}\right)=s_{f} .
$$

As for the SoC, it is bounded at the beginning and the end of the trip, thus (21) and (22) are completed with $\xi(0)=\xi_{i}$ for $j=1$ and $\xi\left(t_{f}\right)=\xi_{f}$ for $j=n_{b}$, respectively.

\section{Offline Solutions}

Two contexts for solving the ED-OCP are recognizable, namely, offline and online. An offline solution assumes that all road characteristics and positiondependent constraints are known in advance. In contrast, an online solution 
is a real-time-capable solution that makes use of predictions and estimations that are typically available on a vehicle immersed in its environment. Another difference is in computation time, since online solutions must be compatible with real-time execution.

In the following sections, the three solution methods for offline optimization that are the most relevant for automotive engineers are discussed, namely, Pontryagin's minimum principle (PMP), dynamic programming (DP), and analytical solutions. Fully parametric solutions [36, 38, genetic algorithmes [46], and semi-heuristic approaches, including the parametric optimization [29, 30] and the optimal tracking [31] of assumed speed profiles, are not discussed further.

\section{Pontryagin's Minimum Principle}

Pontryagin's minimum principle [47] is the basis for the analytical solutions described below. The optimal solution of the ED-OCP is calculated after having defined the Hamiltonian function as

$$
H(u, x, \lambda)=g(u, x)+\lambda^{T} f(u, x),
$$

with $\lambda$ being a vector adjoint to the state vector $x$. Let $u^{*}(t)$ be an optimal control policy and $x^{*}(t), \lambda^{*}(t)$ the resulting state and adjoint state trajectories, respectively. Then

$$
\begin{gathered}
\dot{x}^{*}(t)=\frac{\partial H}{\partial \lambda}\left(x^{*}(t), u^{*}(t), \lambda^{*}(t)\right), \quad x^{*}(0)=x_{0}, \quad x^{*}\left(t_{f}\right)=x_{f}, \\
\dot{\lambda}^{*}(t)=-\frac{\partial H}{\partial x}\left(x^{*}(t), u^{*}(t), \lambda(t)^{*}\right), \\
u^{*}(t)=\arg \min _{u \in U} H\left(x^{*}(t), u, \lambda(t)^{*}\right) .
\end{gathered}
$$

Note that (26) is the formulation of the "minimum principle" 48. When $H$ is linear in the control variables, the optimal solution requires the control variables to be at one point or another of the boundary of the feasible control region $U$ ("bang-bang" control) [47]. In such circumstances, the function $\sigma=\partial H / \partial u$ is called the "switching function" because a change of its sign makes the corrisponding control variable switch from one point on the boundary of $U$ to another point on the boundary. The case where $\sigma \equiv 0$ in a finite interval results in a singular arc.

The system of differential equations (25) is not easily solved because the initial conditions on the adjoint vector $\lambda^{*}$ are not given. Instead, the state vector $x^{*}$ is subject to boundary conditions both at the initial time 
and at the final time. This problem is a two-point boundary-value problem (TPBVP), which may be solved using time-consuming multiple shooting algorithms [41. Moreover, state constraints introduce additional unknowns (jump conditions) that have to be determined as well. Overall, numerically solving of the TPBVP is sensitive to the shooting algorithm adopted and thus its direct use is rather inpractical both for offline and online applications.

\section{Dynamic Programming}

Numerical offline methods such as dynamic programming and Dijkstralike algorithms [41] are widely used to solve the ED-OCP. In particular, DP [49, 50] is applied both with time as the independent variable with $t_{f}$ fixed and, in position domain, with $s_{f}$ fixed [3, 18, 21, 24, 28, 33, 35, 37]. DP solves a discretized version of the ED-OCP (18)-(22), therefore it requires a discretization of the independent variable into $N$ steps, together with a discretization of the state and control spaces. Using the forward Euler scheme with a $1 \mathrm{~s}$ time step, the discretized ED-OCP can be formulated as

$$
\min _{u_{k} \in U} J=\sum_{k=1}^{N} g_{k}\left(u_{k}, x_{k}\right),
$$

subject to state dynamics,

$$
x_{k+1}=f_{k}\left(u_{k}, x_{k}\right),
$$

with state and control constraints obtained from (20)-(22).

For ICEs and EVs, the control space is limited to $u=\left\{F_{w}, F_{b}\right\}$ and the state space to $x=\{s, v\}$. However, since the computation time of the DP solution grows exponentially with the number of states, it is desirable to further reduce the size of the problem when possible. A state reduction can be performed when perturbations and constraints depend on time, or position, but not on both. This case applies to highway and urban scenarios without traffic lights, where perturbations (for instance, distance-depending slopes) and constraints (for instance, top speed limits when not variable according to the traffic congestion and/or air pollution) depend on position but not explicitly on time. In such scenarios, position can be used as the independent variable using the transformation (14), and speed as the only state variable. The constraint on the final position is fulfilled by construction. Final time constraint, however, has to be enforced separately. For that, an additional tunable term is added to the cost function as a terminal cost 
$\beta \cdot t_{f}[18,24,37]$. If the position space is discretized in $N$ steps, $s_{k}=k \Delta s$, $k=0, \ldots, N, t_{f}=\sum \Delta t_{k}$, and $\Delta t_{k}=\Delta s / \bar{v}_{k}$, with

$$
\bar{v}_{k}=\frac{v_{k}+v_{k+1}}{2}
$$

the penalty term can be transferred to the running cost that becomes

$$
g_{k}\left(u_{k}, x_{k}\right)=\left(P_{\operatorname{tank}}\left(u_{k}, \bar{v}_{k}\right)+\beta\right) \frac{\Delta s}{\bar{v}_{k}} .
$$

To determine the right value of the tunable coefficient $\beta$, a root-finding method is used to drive the final time error to zero [18, 24, 37.

The minimal cost to reach the final state from a given state $x_{k}$ is the cost-to-go function $J_{k}\left(x_{k}\right)$,

$$
J_{k}\left(x_{k}\right)=\min _{u_{k} \in U_{k}\left(x_{k}\right)}\left\{g_{k}\left(u_{k}, x_{k}\right)+J_{k+1}\left(f_{k}\left(u_{k}, x_{k}\right)\right)\right\}, \quad k=1, \ldots, N-1 .
$$

The DP algorithm paradigm now states that, if $u_{k}^{*}\left(x_{k}\right)$ minimizes the righthand side of (31) for each $x_{k}$ and $k$, the control policy $\left\{u_{1}^{*}, \ldots, u_{N}^{*}\right\}$ is optimal. Starting at $k=N-1$ with an inialization of $J_{N}=g_{N}\left(x_{N}\right)$ and going backwards, the minimal cost-to-go can recursively be calculated for each $x_{k}$ and $k$, which leads to the optimal state trajectory and the optimal control policy.

\section{Bi-level Optimization}

For HEVs, a third state variable would be necessary, that is, SoC $\xi$. As an alternative to simultaneous optimization, a bi-level approach allows a reduction of computation time [45]. In this approach, the optimal control policy $u$ is found by decoupling its components. In the outer loop the speed trajectory is optimized with respect to the control vector $u^{(1)}:=\left\{F_{w}, F_{b}\right\}$ only, thus as for an ICE or an EV. The running cost of this subproblem is found by solving a second subproblem (inner loop), where the power split is optimized $\left(u^{(2)}:=\left\{T_{m}\right\}\right)$ for a given state and wheel force, while $T_{e}$ is found from (44). An overview of the algorithm is given in Figure 3 .

In a convenient embodiement of the method [45], the inner loop can be performed with the PMP-based technique known in the HEV control literature as equivalent consumption minimization strategy (ECMS) [1]. The Hamiltonian of this subproblem is defined as

$$
H\left(T_{m}, u^{(1)}, v\right)=P_{f}\left(T_{m}, u^{(1)}, v\right)+\lambda_{\xi} \cdot P_{b}\left(T_{m}, u^{(1)}, v\right) .
$$


The costate adjoint to the $\mathrm{SoC}, \lambda_{\xi}$, is found such that it enforces the constraint over the final SoC (22). Hence, the powertrain torque provided by the motor is a function of only vehicle speed and vehicle acceleration,

$$
T_{m}^{*}\left(u^{(1)}, v\right)=\arg \min _{T_{m}} H\left(T_{m}, u^{(1)}, v\right) .
$$

As a consequence, the fuel consumption is dependent on only those two variables and is denoted as $P_{f}^{*}\left(u^{(1)}, v\right)$.

In the outer loop, DP is used with the position as the independent variable and the running cost

$$
g_{k}\left(u_{k}, x_{k}\right)=\left(P_{f}^{*}\left(F_{w, k}, \bar{v}_{k}\right)+\beta\right) \frac{\Delta s}{\bar{v}_{k}} .
$$

As described above, the coefficient $\beta$ is tuned in an iterative process as to enforce the constraint over final time.

\section{Analytical Solutions}

Analytical solutions avoid sensitivity problems of PMP and are more suitable than DP for online solving of the ED-OCP due to reduced computation time. These techniques are still based on equations (23)-(26), which are solved in a closed form. For this purpose, they require a sufficiently simple modeling of the system (that is, functions $g$ and $f$, as well as constraints).

Analytical solutions have been derived for EVs [28, FCEVs [14, ICEVs [19, 22], also with regenerative braking capability [51], and HEVs [23]. To illustrate various solution approaches, each powertrain is separately treated in the following sections.

\section{Analytical Solutions for Electric Vehicles}

The modeling assumptions used here, representing both battery EVs and fuel cell EVs, are: (i) $\alpha=$ constant, (ii) $F_{\text {res }}=m g c_{r}+m g \sin (\alpha)$, (iii) $b_{0}=b_{0}^{\prime}=b_{1}^{\prime}=0$, (iv) $R_{b}=0$, (v) $u=\left\{T_{m}, F_{b}\right\}$, (vi) $T_{m, m i n}=$ constant, $T_{m, \max }=$ constant.

Under these assumptions, the state function is

$$
f(u(t), x(t))=\left(\begin{array}{c}
\dot{s} \\
\dot{v}
\end{array}\right)=\left(\begin{array}{c}
v \\
h_{1} T_{m} \eta_{t}^{\operatorname{sign}\left(T_{m}(t)\right)}-d_{0}-w
\end{array}\right),
$$

where

$$
h_{1}:=\frac{\gamma_{m}}{m r_{w}}, \quad d_{0}:=\frac{F_{\mathrm{res}}}{m}, \quad w:=\frac{F_{b}}{m} .
$$


The running cost function $g$ is

$$
g=b_{1} T_{m} v+b_{2} T_{m}^{2}
$$

The Hamiltonian is

$$
H=b_{1} T_{m} v+b_{2} T_{m}^{2}+\lambda_{v}\left(h_{1} T_{m} \eta_{t}^{\operatorname{sign}\left(T_{m}(t)\right)}-d_{0}-w\right)+\lambda_{s} v
$$

where $\lambda_{v}$ and $\lambda_{s}$ are the two adjoint states. The application of PMP yields the control law (for simplicity, the asterisk is omitted except from the optimal control inputs)

$$
T_{m}^{*}(t)=\left\{\begin{array}{lll}
\min \left(T_{m, \max }, T_{m}^{+}(t)\right) & \text { if } \quad T_{m}^{+}(t)>0, T_{m}^{-}(t)>0, \\
0 & \text { if } \quad T_{m}^{+}(t)<0, T_{m}^{-}(t)>0, \\
\max \left(T_{m, \min }, T_{m}^{-}(t)\right) & \text { if } \quad T_{m}^{+}(t)<0, T_{m}^{-}(t)<0,
\end{array}\right.
$$

where $T_{m}^{+}(t):=-\frac{1}{2 b_{2}}\left(b_{1} v(t)+h_{1} \eta_{t} \lambda_{v}(t)\right), T_{m}^{-}(t):=-\frac{1}{2 b_{2}}\left(b_{1} v(t)+\frac{h_{1} \lambda_{v}(t)}{\eta_{t}}\right)$, and

$$
w^{*}(t)=\left\{\begin{array}{lll}
0 & \text { if } \quad \lambda_{v}(t)<0 \\
W & \text { if } \quad \lambda_{v}(t)>0
\end{array}\right.
$$

The adjoint dynamics are

$$
\dot{\lambda}_{v}(t)=-b_{1} T_{m}^{*}(t)-\lambda_{s}(t), \quad \dot{\lambda}_{s}(t)=0 .
$$

The analytical solution (39)-(40) shows that there are six possible optimal modes of operation:

- Maximal Acceleration (SA): $T_{m}^{+}>T_{m, \max }, T_{m}^{-}>0, \lambda_{v}<0$,

- Acceleration (A): $0<T_{m}^{+}<T_{m, \max }, T_{m}^{-}>0, \lambda_{v}<0$,

- Coasting (C): $T_{m}^{+}<0, T_{m}^{-}>0, \lambda_{v}<0$,

- Deceleration (D): $T_{m}^{-}<0, T_{m, \min }<T_{m}^{-}<0, \lambda_{v}<0$,

- Maximal Deceleration (SD): $T_{m}^{+}<0, T_{m}^{-}<T_{m, \min }, \lambda_{v}<0$,

- Braking (B): $T_{m}^{+}<0, T_{m}^{-}<T_{m, \min }, \lambda_{v}>0$,

while the mode $\lambda_{v}>0, T_{m}^{-}>T_{m, \min }$ would be physically counter-intuitive. Note that mode $\mathrm{C}$ appears due the presence of the term $\eta_{t}^{\operatorname{sign}\left(T_{m}\right)}$ in the Hamiltonian function and, for the same reason, mode $\mathrm{A}$ is distinguished from mode D. 
The particular sequence that builds up the optimal solution depends on the boundary conditions. Assuming a sequence, for instance, SA-A-CD-SD-B, see Figure 4, the calculation of the optimal velocity and position trajectories can be performed in a sequential fashion.

Start with unknown $\lambda_{v}(0), \lambda_{s}$, and integrate (39)-(41) for each phase of the sequence. Switching times between phases $t_{i}, i=1, \ldots, 5$ are defined by the switching conditions $T_{m}^{+}\left(t_{1}\right)=T_{m, \max }, T_{m}^{+}\left(t_{2}\right)=0, T_{m}^{-}\left(t_{3}\right)=0$, $T_{m}^{-}\left(t_{4}\right)=T_{m, \min }, \lambda_{v}\left(t_{5}\right)=0$. Then impose the two terminal conditions $v_{f}\left(t_{f}\right)=v_{f}$ and $s\left(t_{f}\right)=s_{f}$ and obtain two equations $f_{1}\left(\lambda_{v}(0), \lambda_{s}\right)=0$ and $f_{2}\left(\lambda_{v}(0), \lambda_{s}\right)=0$ in the two unknown initial conditions. It turns out that the former equation is affine in the variable $\lambda_{v}(0)$. By solving and replacing this variable in the latter equation, a tenth-order polynomial equation $f_{3}\left(\lambda_{s}\right)=0$ is obtained, which has only one physically meaningful solution.

If further simplifications are assumed, that is: (i) $\eta_{t}=1$, (ii) $W=0$ (no friction braking), then $T_{m}^{+}(t) \equiv T_{m}^{-}(t)$ and the three-phase solution presented in [14] (for variable $\alpha$ ) is obtained. If, additionally, (iii) $T_{m, \max }=$ $-T_{m, \min } \rightarrow \infty$, then there is only one phase left. The resulting control trajectory $T_{m}^{*}(t)$ can be explicitly calculated and it appears to be an affine function of time, while the optimal trajectory $v^{*}(t)$ is a quadratic function of time [28, 52 ]

$$
v^{*}(t)=v_{0}-\frac{4 v_{i} t}{t_{f}}-\frac{2 v_{f} t}{t_{f}}-\frac{6 s_{f} t^{2}}{t_{f}^{3}}+\frac{6 s_{f} t}{t_{f}^{2}}+\frac{3 v_{i} t^{2}}{t_{f}^{2}}+\frac{3 v_{f} t^{2}}{t_{f}^{2}} .
$$

\section{Analytical Solutions for Internal Combustion Engine Vehicles}

The modeling assumptions used here are: (i) $\eta_{t}=1$, (ii) $\gamma_{e}(t)=\gamma=$ constant, (iii) $\alpha=$ constant, (iv) $F_{\text {res }}(t)=f_{0}+f_{1} v(t)$, (v) $P_{f, 0}(t)=\omega_{e}(t) T_{f, 0}$, (vi) $e=$ constant, (vii) $u=\left\{T_{e}, F_{b}\right\}$.

Under these assumptions, the state function is

$$
f(u(t), x(t))=\left(\begin{array}{c}
\dot{s} \\
\dot{v}
\end{array}\right)=\left(\begin{array}{c}
v \\
h_{1} T_{e}-d_{0}-d_{1} v-w
\end{array}\right),
$$

where $h_{1}$ and $w$ are defined as in (36), while $d_{0}:=\frac{f_{0}}{m}$ and $d_{1}:=\frac{f_{1}}{m}$. From (86) and (18), the cost function is composed of two terms, with the latter being proportional to the integral of $\omega_{e}$. Since this quantity is assigned to be equal to $s_{f}$, the second term of the cost function is a constant. Consequently, the relevant running cost $g$ is

$$
g=\frac{T_{e}}{e} \omega_{e}=a_{1} T_{e} v
$$


with $a_{1}:=\frac{\gamma}{r_{w} e}$. The Hamiltonian is

$$
H=a_{1} T_{e} v+\lambda_{v}\left(h_{1} T_{e}-d_{0}-d_{1} v-w\right)+\lambda_{s} v,
$$

where $\lambda_{v}$ and $\lambda_{s}$ are the two adjoint states. The application of PMP yields the engine control law

$$
T_{e}^{*}(t)=\left\{\begin{array}{lll}
T_{e, \max }(v(t)) & \text { if } & \sigma(t)<0 \\
T_{\sigma} & \text { if } & \sigma(t)=0, \\
T_{e, \min }(v(t)) & \text { if } & \sigma(t)>0
\end{array}\right.
$$

where the switching function $\sigma(t):=\partial H / \partial T_{e}=a_{1} v(t)+h_{1} \lambda_{v}(t), T_{\sigma}$ is the optimal torque in the singular case, and

$$
w^{*}(t)=\left\{\begin{array}{lll}
0 & \text { if } & \lambda_{v}(t)<0 \\
W & \text { if } & \lambda_{v}(t)>0
\end{array}\right.
$$

where $W:=F_{b, \max } / m$. The adjoint dynamics are

$$
\dot{\lambda}_{v}(t)=-a_{1} T_{e}^{*}(t)+d_{1} \lambda_{v}(t)-\lambda_{s}(t), \quad \dot{\lambda}_{s}(t)=0 .
$$

From the conditions $\sigma=0$ and $\dot{\sigma}=0$ that define a singular arc, such arc is shown to be a constant-speed trajectory [53]. Consequently, $T_{\sigma}$ is constant on a singular arc. Therefore, the analytical solution (46)-(47) shows that there are four possible optimal modes of operation:

- Acceleration (A): $\sigma<0, \lambda_{v}<0$,

- Constant Speed (C): $\sigma=0, \lambda_{v}<0$,

- Deceleration (coasting, D): $\sigma>0, \lambda_{v}<0$,

- Braking (B): $\sigma>0, \lambda_{v}>0$.

For small values of $d_{1}$, it can be shown that both $\sigma$ and $\lambda$ are affine functions of time and thus can have at most one sign switch. Therefore, only four basic sequences of control modes are possible: A-C-D-B, A-C-A, D-C-D-B or DC-A. The particular sequence that builds up the optimal solution depends on the boundary conditions. For example, the sequence A-C-D-B is likely to be optimal when both $v_{i}$ and $v_{f}$ are small or zero; see Figure 5 .

Contrarily to the EV case, the sequential integration of (43), (46)-(48) as a function of the unknown initial values $\lambda_{v}(0)$ and $\lambda_{s}(0)$ is not possible since a singular arc $(\mathrm{C})$ always appears in the optimal sequence. However, 
the ED-OCP can be reduced into a parametric optimization problem, where the correct sequence of control modes and the switching times between these modes, $t_{i}, i=1, \ldots, 3$ are to be found. For three-phase mode sequences the two switching times $t_{1}$ and $t_{2}$ are fully defined by the terminal conditions $v\left(t_{f}\right)=v_{f}, s\left(t_{f}\right)=s_{f}$. For four-phase mode sequences one degree of freedom among the three switching times is left. This degree of freedom is found in such a way to minimize the fuel consumption.

The parametric optimization method can be extended to consider less restrictive assumptions than those listed above, for instance, variable gear ratio or engine torque limits variable with speed. A possible embodiment is presented in "Parametric Optimization Method for ICEVs".

\section{Analytical Solutions for Hybrid Vehicles}

The modeling assumptions used here (parallel hybrid) are: (i) $\eta_{t}=1$, (ii) $\gamma_{e}(t)=\gamma_{m}=\gamma=\mathrm{const}$, (iii) $\alpha=$ constant, (iv) $F_{\text {res }}(t)=f_{0}+f_{1} v(t)$, (v) $P_{f, 0}(t)=\omega_{e}(t) T_{f, 0}$, (vi) $e=$ constant, (vii) $b_{0}=b_{0}^{\prime}=b_{1}^{\prime}=0$, (viii) $R_{b}=0$, (ix) $u=\left\{T_{e}, T_{m}, F_{b}\right\}$.

Under these assumptions, the state function is

$$
f(u(t), x(t))=\left(\begin{array}{c}
\dot{s} \\
\dot{v} \\
\dot{\xi}
\end{array}\right)=\left(\begin{array}{c}
v \\
h_{1}\left(T_{m}+T_{e}\right)-d_{0}-d_{1} v-w \\
-b_{1} v T_{m}-b_{2} T_{m}^{2}
\end{array}\right),
$$

while, similarly to the ICEV case, the running cost is

$$
g=a_{1} T_{e} v,
$$

with the usual definition of the parameters. The Hamiltonian is

$$
H=a_{1} T_{e} v+\lambda_{v}\left(h_{1}\left(T_{m}+T_{e}\right)-d_{0}-d_{1} v-w\right)+\lambda_{s} v-\lambda_{\xi}\left(b_{1} v T_{m}+b_{2} T_{m}^{2}\right) .
$$

The application of PMP yields the control law

$$
T_{m}^{*}(t)=\left\{\begin{array}{lll}
T_{m, \max }(v(t)) & \text { if } \quad T_{m}^{o}(t)>T_{m, \max }(v(t)), \\
T_{m}^{o}(t) & \text { if } T_{m, \min }(v(t)) \leq T_{m}^{o}(t) \leq T_{m, \max }(v(t)), \\
T_{m, \min }(v(t)) & \text { if } T_{m}^{o}(t)<T_{m, \min }(v(t)),
\end{array}\right.
$$

where $T_{m}^{o}(t):=\frac{\left(h_{1} \lambda_{v}(t)-b_{1} \lambda_{\xi}(t) v(t)\right)}{2 b_{2} \lambda_{\xi}(t)}$

$$
T_{e}^{*}(t)=\left\{\begin{array}{lll}
T_{e, \max }(v(t)) & \text { if } & \sigma(t)<0 \\
T_{\sigma} & \text { if } & \sigma(t)=0 \\
T_{e, \min }(v(t)) & \text { if } & \sigma(t)>0
\end{array}\right.
$$


where $\sigma(t):=a_{1} v(t)+h_{1} \lambda_{v}(t)$, and

$$
w^{*}(t)=\left\{\begin{array}{lll}
0 & \text { if } \quad \lambda_{v}(t)<0 \\
W & \text { if } \quad \lambda_{v}(t)>0 .
\end{array}\right.
$$

The adjoint dynamics are

$\dot{\lambda}_{v}(t)=-a_{1} T_{e}(t)-a_{1} T_{m}(t)+d_{1} \lambda_{v}(t)+\lambda_{s}-b_{1} T_{m}(t)\left(1-\lambda_{\xi}\right), \quad \dot{\lambda}_{s}(t)=0, \quad \dot{\lambda}_{\xi}(t)=0$.

Similarly to the ICEV case, from the conditions $\sigma=0$ and $\dot{\sigma}=0$, the singular arc is shown to be a constant-speed trajectory. Additionally, the condition $\lambda_{v}>0$ implies that $T_{m}^{o}<0$ and $\sigma>0$. Therefore, the solution (52) -(55) shows that there are several possible optimal modes of operation, including:

- Maximal hybrid acceleration (AH), $\sigma<0, \lambda_{v}<0, T_{m}^{o} \geq T_{m, \max }$,

- Maximal electric acceleration (AE), $\sigma>0, \lambda_{v}<0, T_{m}^{o} \geq T_{m, \max }$,

- Maximal regenerative braking (DE), $\sigma>0, \lambda_{v}<0, T_{m}^{o} \leq T_{m, \min }$,

- Maximal hybrid recharge $(\mathrm{RH}), \sigma<0, \lambda_{v}<0, T_{m}^{o} \leq T_{m, \min }$,

- Braking and regenerative braking (B), $\sigma>0, \lambda_{v}>0, T_{m}^{o} \leq T_{m, m i n}$,

- Optimum hybrid operation $(\mathrm{H}), \sigma<0, \lambda_{v}<0, T_{m, \min } \leq T_{m}^{o} \leq$ $T_{m, \max }$,

- Optimum electric operation (E), $\sigma>0, \lambda_{v}<0, T_{m, \min } \leq T_{m}^{o} \leq$ $T_{m, \max }$

- Constant speed (C), $\sigma=0, \lambda_{v}<0$.

Two additional modes, the coasting mode (D) and the full ICE mode (A), appear when assumption (i) is relaxed. Conversely, if the further simplification $b_{2}=0$ is assumed, the bang-bang solution of [23] is obtained, where only the first five modes are possible.

\section{Example Simulation Results}

Three examples of offline-calculated solutions of the ED-OCP are presented in this section. DP results are analyzed first and then compared with analytical solutions. While the former are based on accurate tabulated models for 
the engine (7), the motor (9), and the transmission (15), analytical solutions are based on the simplified equations presented in the corresponding sections above. Consequently, differences in evaluating fuel consumption arise and are presented below.

Figure 6 shows position, speed, motor torque, and battery energy for an $\mathrm{EV}$, as calculated with a DP algorithm for two sets of boundary conditions. The first case $\left(s_{f}=200 \mathrm{~m}, t_{f}=24 \mathrm{~s}\right)$ shows the six phases described above (SA-A-C-D-SD-B). The solution for the second case $\left(s_{f}=100 \mathrm{~m}\right.$, $t_{f} \approx 12 \mathrm{~s}$ ) shows a longer phase SA and virtually no phases $\mathrm{C}$ and $\mathrm{D}$. Similar results are obtained with the sequential method presented above. A comprehensive comparison between DP, the sequential analytical solution, and the quadratic explicit solution is presented in [28, where it is shown that the average error in predicting the minimal energy consumption over the range $d_{f}=50-500 \mathrm{~m}$ and $d_{f} / t_{f}=10-50 \mathrm{~km} / \mathrm{h}\left(\right.$ with $\left.v_{i}=v_{f}=\alpha=0\right)$ is less than $1 \%$ with the sequential method, and about $5 \%$ with the explicit method.

Example offline solutions for an ICEV are given in Figures 748, which show position, speed, engine torque, and fuel energy, as calculated with a DP algorithm for four sets of boundary conditions. The first case $s_{f}=250$ $\left.\mathrm{m}, t_{f} \approx 30 \mathrm{~s}\right)$ shows the mode sequence A-C-D-B. The second case $\left(s_{f}=150\right.$ $\left.\mathrm{m}, t_{f}=18 \mathrm{~s}\right)$ has no phase $\mathrm{C}$. In the third case $\left(s_{f}=250 \mathrm{~m}, t_{f} \approx 26 \mathrm{~s}\right)$, the sequence is A-C-A. In the fourth case $\left(s_{f}=250 \mathrm{~m}, t_{f}=30 \mathrm{~s}\right)$, the sequence is D-C-D. Note the torque discontinuities during A and D phases due to gear changes. Since the analytical solution (46)-(47) assumes constant gear, the differences between DP and the analytical solution tend to be larger than in the EV case (more than $15 \%$ for a typical midsized car). However, using the parametric optimization method presented in the "Parametric Optimization Method for ICEVs," differences are reduced to less than 2\% (Figure 9).

An example offline solution for an HEV is shown in Figure 10 in terms of vehicle speed and torque from engine and motor, as calculated with DP for a sequence of seven segments between two vehicle stops. The figure shows various mode sequences for distinct segments, namely, AH-C-D (first segment), AH-C-D-B (second, fourth, and sixth segment), AH-C-B (third and seventh segment), and AH-D-B (fifth segment). 


\section{Online Application and Known Experimental Results}

Only a few of the approaches presented above have been experimentally demonstrated by an onboard implementation. While the demonstrated systems will probably be incorporated in fully automated driving applications [54, so far all of them interact with a human driver (online assessment systems, online advice systems) or have been tested as a replacement of conventional cruise controllers (predictive cruise controllers). To the best knowledge of the authors, none of the former approaches tries to include the driver's dynamics into the ED-OCP. In contrast, it is either assumed that the driver will follow (in online advice systems) or that he/she would have followed (in online assessment systems) the optimal speed profiles calculated.

\section{Online Assessment}

The analytical solution method has been experimentally demonstrated for EVs [28]. The methods used are either the explicit solution (42) or the complete six-phase analytical solution implemented with the aid of a neural network, with an input vector $I=\left\{t_{f}, s_{f}, v_{i}, v_{f}\right\}$ and an output vector $O=\left\{t_{1}, \ldots, t_{5}\right\}$, where the $t_{i}$ are the switching times between subsequent phases.

This implementation is part of a generic online assessment system (see classification of ADAS in the first section of this article) that is operational for EVs and ICEs [55]. For the latter, the parametric optimization technique detailed in the "Parametric Optimization Method for ICEVs" is adopted. While the structure of the solution is calculated using the simplifying assumptions outlined in the "Analytical Solutions" section, the parametric optimization method allows more realistic models to calculate the correct sequence of control modes and the respective switching times.

An optimization run is performed at each breakpoint detected, that is, after each segment completed by the driver. The system then provides an "eco-driving" score, defined as

$$
\operatorname{EDS}:=10\left(2-\frac{E_{b}}{E_{b}^{*}}\right)
$$

where $E_{b}$ is the actual battery energy consumed during the segment, while $E_{b}^{*}$ is the optimum calculated.

The driver is then expected to learn from subsequent scores obtained. Tests conducted on a Peugeot EV have shown a reduction of $14 \%$ in energy 
consumption, an increase of $6 \%$ in the score, with only a slight decrease $(2 \%)$ of the average speed; see Figure 11 and [28].

\section{Online Advice and Predictive Cruise Control}

Online advice EDS and predictive cruise controllers have been demonstrated based on online solving of a DP [17, 18, 26] or a parametric optimization algorithm [20. These approaches are suitable for highway or extra-urban scenarios due to long trip duration and low control frequency. Numerical improvements to standard DP techniques are essential in this respect [56].

Tests conducted on a Scania tractor on a highway route have shown overall fuel consumption reductions up to $6 \%$ (depending on the set cruise speed), with time increase lower than $2 \%$ [18. The approach has been demonstrated also for two Porsche cars on an extra-urban route, resulting in $24 \%$ and $10 \%$ reduction of the fuel consumption, respectively, with a reduction of the average speed below $2 \%$ in both cases [26]. Tests of a similar concept on a chassis dynamometer have shown an average fuel consumption reduction of about $5 \%$ [20].

\section{Conclusions}

This article has presented a general formulation of energy-efficient driving of road vehicles as an optimal control problem. Several scenarios have been considered in the general framework and the most-adopted solution techniques have been presented. The article also presents some advancements in real-time computing of the optimal speed profiles, particularly for ICEVs and HEVs. For ICEVs, a parametric optimization technique inspired by the analytical solution of a simplified version of the ED-OCP has been illustrated. For HEVs, a bi-level algorithm that tries to decouple energy-optimal drive control from hybrid energy-management control has been presented. The article has also discussed a few experimental results published. As this section shows, experimental demonstration of the "optimal in-trip advice" concept seems to be limited so far to highway and extra-urban scenarios, mainly as an extension of a cruise control system. For urban-like situations, only the "optimal in-trip assessment" has been demonstrated.

Future research will be focused on the robustification of online calculations for all types of powertrains, and the generalization of the optimal in-trip advice to all possible scenarios, including urban driving. Interactions with the infrastructure will be integrated in the system. In particular, opti- 
mal approach to traffic lights and green waving will be made possible thanks to a dedicated vehicle-to-infrastructure communication. 


\section{References}

[1] L. Guzzella and A. Sciarretta, Vehicle Propulsion Systems, Berlin: Springer, third edition, 2013.

[2] O. Sundström and C. Binding, "Optimization methods to plan the charging of electric vehicle fleets", ACEEE Int. Journal on Communication, vol. 1, no. 2, pp. 45-50, 2010.

[3] B. Seraens, "Optimal control based eco-driving," Doctorate thesis, Katholieke Universiteit Leuven, 2012. Available at WWW.researchgate.net.

[4] P. Seewald, J. Josten, A. Zlocki, and L. Eckstein, "User acceptance evaluation approach of energy efficient driver assistance systems," Proc. 9th ITS European Congress (ITSEC), Dublin, Ireland, 2013.

[5] T. Hof, L. Conde, E. Garcia, A. Iviglia, S. Jamson, A. Jopson, F. Lai, N. Merat, J. Nyberg, S. Rios, D. Sanchez, S. Schneider, P. Seewald, C. van der Weerdt, R. Wijn, A. Zlocki, "A state of the art review and users' expectations", European Commission FP7 project Ecodriver, 2014. Available at ww. ecodriver-project.eu.

[6] R.H. Goddard, "A method for reaching extreme altitudes," Smithsonian Miscellaneous Collections, vol. 71, no. 2, 1919.

[7] A. Miele, "Optimum climbing technique for a rocket-powered aircraft," Jet Propulsion, vol. 25, pp. 385-391, 1955.

[8] H. Munick, "Goddard problem with bounded thrust," AIAA Journal, vol. 3, no. 7, pp. 1283-1285, 1965.

[9] P. Tsiotras and H.J. Kelley, "Drag-law effects in the Goddard problem," Automatica, vol. 27, no. 3, pp. 481-490, 1991.

[10] K. Graichen, N. Petit, "Solving the Goddard problem with thrust and dynamic pressure constraints using saturation functions," Prov. of the 17th IFAC World Congress, Seoul, Korea, 2008, pp. 14301-14306.

[11] V. Nezhadali and L. Eriksson, "Wheel loader optimal transients in the short loading cycle," Proc. 19th IFAC World Congress, Cape Town, South Africa, 2014, pp. 7917-7922. 
[12] D. Chang and E. Morlok, "Vehicle speed profiles to minimize work and fuel consumption," J. Transp. Eng., vol. 131, no. 3, pp. 173-182, 2005.

[13] L. Nouveliére, M. Braci, L. Menhour, H.T. Luu, and S. Mammar, "Fuel consumption optimization for a city bus," Proc. UKACC Control Conf., Manchester, UK, 2008.

[14] A. Sciarretta, L. Guzzella, and J. van Baalen, "Fuel optimal trajectories of a fuel cell vehicle," Proc. IFAC Int. Conf. on Advances in Vehicle Control and Safety (AVCS), Genoa, Italy, 2004.

[15] J.-J. Santin (ed.), The World's Most Fuel Efficient Vehicle: Design and Development of Pac-Car II, Zurich: vdf Hochschulverlag AG an der ETH Zürich, 2007.

[16] A. Stoicescu, "On fuel-optimal velocity control of a motor vehicle," Int. J. of Vehicle Design, vol. 17, no. 2/3, pp. 229-256, 1995.

[17] F. Lattemann, K. Neiss, S. Terwen, and T. Connolly, "The Predictive Cruise Control - A system to reduce fuel consumption of heavy duty trucks," SAE Technical Paper no. 2004-01-2616, 2004.

[18] E. Hellström, M. Ivarsson, J. Åslund, and L. Nielsen, "Look-ahead control for heavy trucks to minimize trip time and fuel consumption," Control Engineering Practice, vol. 17, no. 2, pp. 245-254, 2009.

[19] B. Passenberg, P. Kock, and O. Stursberg, "Combined time and fuel optimal driving of trucks based on a hybrid model," Proc. 10th European Control Conference, Budapest, Hungary, 2009, pp. 4955-4960.

[20] T. van Keulen, B. de Jager, D. Foster, and M. Steinbuch, "Velocity trajectory optimization in hybrid electric trucks," Proc. American Control Conference, Baltimore, MD, 2010, pp. 5074-5079.

[21] G. Heppeler, M. Sonntag, and O. Sawodny, "Fuel efficiency analysis for simultaneous optimization of the velocity trajectory and the energy management in hybrid electric vehicles," Proc. 19th IFAC World Congress, Cape Town, South Africa, 2014.

[22] E. Ozatay, U. Ozguner, J. Michelini, and D. Filev, "Analytical solution to the minimum energy consumption based velocity profile optimization problem with variable road grade," Proc. 19th IFAC World Congress, Cape Town, South Africa, 2014. 
[23] T.S. Kim, C. Manzie, and R. Sharma, "Two-stage optimal control of a parallel hybrid vehicle with traffic preview." Proc. 18th IFAC World Congress, Milano, Italy, 2011, pp. 2115-2120.

[24] W. Dib, L. Serrao, and A. Sciarretta, "Optimal control to minimize trip time and energy consumption in electric vehicles.", Proc. IEEE Vehicle Power and Propulsion Conf. (VPPC), Chicago, IL, 2011, pp. 1-8.

[25] W. Dib, A. Chasse, D. di Domenico, P. Moulin, and A. Sciarretta, "Evaluation of the energy efficiency of a fleet of electric vehicles for eco-driving application," Oil \& Gas Science and Technology - Rev. IFP Energies nouvelles, vol. 67, no. 4, pp. 589-599, 2012.

[26] T. Radke, "Energieoptimale Längsführung von Kraftfahrzeugen durch Einsatz vorausschauender Fahrstrategien," Ph.D. Dissertation, Karlsruhe Inst. Tech., 2013. Available at www.ubka.uni-karlsruhe.de.

[27] T. Boehme, F. Held, C. Rollinger, H. Rabba, M. Schultalbers, and B. Lampe, "Application of an optimal control problem to a trip-based energy management for electric vehicles," SAE Technical Paper no. 2013-01-1465, 2013.

[28] W. Dib, A. Chasse, P. Moulin, A. Sciarretta, and G. Corde, "Optimal energy management for an electric vehicle in eco-driving applications," Control Engineering Practice, vol. 29, pp. 299-307, 2014.

[29] H. Rakha and R.K. Kamalanathsharma, "Eco-driving at signalized intersections using V2I communication," Proc. 14th Int. IEEE Conf. on Intelligent Transportation Systems (ITSC), Wasington, DC, 2011, pp. 341-346.

[30] S. Mandava, K. Boriboonsomsin, and M. Barth, "Arterial velocity planning based on traffic signal information under light traffic conditions," Proc. 12th Int. IEEE Conf. on Intelligent Transportation Systems (ITSC), St. Louis, MO, 2009, pp. 1-6.

[31] B. Asadi and A. Vahidi, "Predictive cruise control: Utilizing upcoming traffic signal information for improving fuel economy and reducing trip time," IEEE Trans. on Control Systems Technology, vol. 19, no. 3, pp. 707-714, 2011.

[32] M. Miyatake, M. Kuriyama, and Y. Takeda, "Theoretical study on eco-driving technique for an electric vehicle considering traffic signals," 
Proc. 9th IEEE Int. Conf. on Power Electronics and Drive Systems (PEDS), Singapore, 2011, pp. 733-738.

[33] G. Mahler and A. Vahidi, "Reducing idling at red lights based on probabilistic prediction of traffic signal timings," Proc. IEEE American Control Conf., Montreal, Canada, 2012, pp. 6557-6562.

[34] E. Ozatay, U. Ozguner, D. Filev, and J. Michelini, "Analytical and numerical solutions for energy minimization of road vehicles with the existence of multiple traffic lights," Proc. 52nd IEEE Annual Conf. on Decision and Control (CDC), Firenze, Italy, 2013, pp. 7137-7142.

[35] G. De Nunzio, C. Canudas de Wit, and P. Moulin, "Eco-driving in urban traffic networks using traffic signal information," Proc. 52nd IEEE Annual Conf. on Decision and Control (CDC), Firenze, Italy, 2013, pp. 892-898.

[36] M.A.S. Kamal, M. Mukai, J. Murata, and T. Kawabe, "Model predictive control of vehicles on urban roads for improved fuel economy," IEEE Trans. on Control Systems Technology, vol. 21, no. 3, pp. 831$841,2012$.

[37] F. Mensing, E. Bideaux, R. Trigui, and H. Tattegrain, "Trajectory optimization for eco-driving taking into account traffic constraints," Transportation Research Part D: Transport and Environment, vol. 18, pp. 55-61, 2012.

[38] D. Lang, T. Stanger, and L. del Re, "Opportunities on fuel economy utilizing V2V based drive systems," SAE Technical Paper no. 2013-010985, 2013.

[39] B. Wu, C.-C. Lin, Z. Filipi, H. Peng, and D. Assanis, "Optimal power management for a hydraulic hybrid delivery truck," Vehicle System Dynamics: Int. J. of Vehicle Mechanics and Mobility, vol. 42, no. 1-2, pp. 23-40, 2004.

[40] T. Guan and C. Frey, "EXPert: A driver assistance system for fuel efficient driving," Proc. 3rd Int. Conf. on Machine Control and Guidance (MCG), Stuttgart, Germany, 2012.

[41] D. Grossoleil and D. Meizel, "Practical design of minimal energy controls for an electric bicycle," Proc. 9th Int. Conf. of Modeling, Optimization and Simulation (MOSIM), Bordeaux, France, 2012. 
[42] P. Sahlholm and K.H. Johansson, "Road grade estimation for lookahead vehicle control using multiple measurement runs," Control Engineering Practice, vol. 18, pp. 1328-1341, 2010.

[43] H.K. Fathy, D. Kang, and J.L. Stein, 'Online "vehicle mass estimation using recursive least squares and supervisory data extraction," Proc. American Control Conference, Seattle, WA, 2008, pp. 1842-1848.

[44] E. Koukoumidis, M. Martonosi, and L.-S. Peh, "Leveraging smartphone cameras for collaborative road advisories," IEEE Trans. on Mobile Computing, vol. 11, no. 5, pp. 707-723, 2012.

[45] D.V. Ngo, T. Hofman, M. Steinbuch, and A.F.A. Serrarens, "An optimal control-based algorithm for hybrid electric vehicle using preview route information," Proc. 2010 American Control Conf. (ACC), Baltimore, MD, 2010.

[46] A. Gaier and A. Asteroth, "Evolution of optimal control for energyefficienct transport", Proc. IEEE Intelligent Vehicle Symposium, Dearborn, MI, 2014, pp. 1121-1126.

[47] A.E. Bryson and Y.-C. Ho, Applied Optimal Control: Optimization, Estimation and Control, Washington: Emisphere, 1975.

[48] L.S. Pontryagin, V.G. Boltyanskii, R.V. Gamkrelidze, and E.F. Mishchenko, The Mathematical Theory of Optimal Processes, New York: Interscience, 1962.

[49] R. Bellman and S. Dreyfus, Applied Dynamic Programming, Princeton: Princeton University Press, 1962.

[50] D. Bertsekas, Dynamic Programming and Optimal Control, Belmont: Athena Scientific, vol. I, 1995.

[51] T. van Keulen, A. de Jager, and M. Steinbuch, "Optimal trajectories for vehicles with energy recovery options," Proc. 18th IFAC World Congress, Milano, Italy, 2011.

[52] N. Petit and A. Sciarretta, "Optimal drive of electric vehicles using an inversion-based trajectory generation approach," Proc. 18th IFAC World Congress, Milano, Italy, 2011, pp. 14519-14526.

[53] L. Guzzella and A. Sciarretta, "Fuel optimal control of rendezvous maneuvers for passenger cars," at-Automatisierungstechnik, vol. 53, pp. 244-250, 2005. 
[54] A. Brown, J. Gonder and B. Repac, "An analysis of possible energy impacts of automated vehicle," in Road Vehicle Automation, Lecture Notes in Mobility, G. Meyer and S. Beiker (eds.), Berlin: Springer, 2014, pp. 137-153.

[55] IFP Energies nouvelles, "Geco. Your real-time eco-drive assistant." Available at http://www.geco-drive.fr/.

[56] E. Hellström, J. Åslund, and L. Nielsen, "Design of an efficient algorithm for fuel-optimal look-ahead control," Control Engineering Practice, vol. 18, pp. 1318-1327, 2010.

\section{Sidebar 1: Vehicle Powertrain Architectures}

Considering the onboard energy sources and power converters, modern road vehicles can be classified as internal combustion engine vehicles (ICEVs), electric vehicles (EVs), and hybrid-electric vehicles (HEVs). Other architectures that are emerging, for instance, hybrid-hydraulic vehicles, are not considered here. A general layout of an ICEV and an EV are shown in Figure S1, referring to the powertrain subsystems relavant to this article. A typical engine map, described by equation (77), is shown in Figure [S2, A typical motor map, described by equation (9), is shown in Figure S3.

HEVs can be further classified as parallel, series, or series-parallel (or power-split, or combined) hybrids. All these types have one engine and one electric source, most often an electrochemical battery. Additionally, parallel HEVs have an electric machine mechanically coupled with the engine. Series HEVs have two electric machines, one mechanically coupled with the wheels and the other (generator) mechanically coupled with the engine only, both are electrically coupled with the battery. Finally, series-parallel HEVs have two electric machines, both mechanically coupled with the wheels and the engine, and electrically coupled with the battery. The three general layouts are shown in Figure \$4. 


\section{Sidebar 2: Parametric Optimization Method for ICEVs}

The parametric optimization method implies the preliminary solving of the law of motion (2)-(4) for the modes $\mathrm{A}\left(T_{e}=T_{e, \max }(v), F_{b}=0\right)$, $\mathrm{D}\left(T_{e}=T_{e, \min }(v), F_{b}=0\right)$, and $\mathrm{B}\left(T_{e}=T_{e, \min }(v), F_{b}=m W\right)$, with $\gamma(t)$ variable according to the law (5). Consequently, speed trajectories $v_{A}(\tau), v_{D}(\tau)$, and $v_{B}(\tau)$ are calculated such that $v_{k}(0)=0$, together with related trajectories of position $s_{k}(\tau)=\int_{0}^{\tau} v_{k}(\tau) d \tau$ and fuel consumed $E_{f, k}$, for each $k=\{A, D, B\}$.

For a three-phase sequence, for instance, A-C-A, the optimal speed profile is defined by six parameters: $\tau_{\{1, \ldots, 4\}}, v_{\sigma}, \Delta t$, which are related to the switching times by the relationships $t_{1}=\tau_{2}-\tau_{1}$ and $t_{2}=t_{1}+\Delta t$; see Figure \$5. With the four sequence-depending boundary conditions $v_{A}\left(\tau_{1}\right)=v_{i}, v_{A}\left(\tau_{4}\right)=v_{f}, s_{A}\left(\tau_{4}\right)-s_{A}\left(\tau_{3}\right)+s_{A}\left(\tau_{2}\right)-s_{A}\left(\tau_{1}\right)+v_{\sigma} \Delta t=s_{f}$, $\tau_{4}-\tau_{3}+\tau_{2}-\tau_{1}+\Delta t=t_{f}$ and the two additional conditions $v_{A}\left(\tau_{2}\right)=$ $v_{A}\left(\tau_{3}\right)=v_{\sigma}$, the six parameters can be calculated and the optimal speed profile accordingly.

For a four-phase sequence, for instance, A-C-D-B, the optimal speed profile is defined by eight parameters: $\left\{\tau_{\{1, \ldots, 6\}}, v_{\sigma}, \Delta t\right\}$, which are related to the switching times by the relationships $t_{1}=\tau_{2}-\tau_{1}$, $t_{2}=t_{1}+\Delta t$, and $t_{3}=t_{2}+\tau_{4}-\tau_{3}$; see Figure [S6. The four boundary conditions $v_{A}\left(\tau_{1}\right)=v_{i}, v_{B}\left(\tau_{6}\right)=v_{f}, s_{B}\left(\tau_{6}\right)-s_{B}\left(\tau_{5}\right)+s_{D}\left(\tau_{4}\right)-s_{D}\left(\tau_{3}\right)+$ $s_{A}\left(\tau_{2}\right)-s_{A}\left(\tau_{1}\right)+v_{\sigma} \Delta t=s_{f}, \tau_{6}-\tau_{5}+\tau_{4}-\tau_{3}+\tau_{2}-\tau_{1}+\Delta t=t_{f}$ and the additional conditions $v_{B}\left(\tau_{5}\right)=v_{D}\left(\tau_{4}\right)$ and $v_{A}\left(\tau_{2}\right)=v_{\sigma}=v_{D}\left(\tau_{3}\right)$ add up to seven conditions. Hence, a degree of freedom is remaining. The latter is chosen in such a way to minimize the function

$E_{f}=E_{f, A}\left(\tau_{2}\right)-E_{f, A}\left(\tau_{1}\right)+E_{f, D}\left(\tau_{3}\right)-E_{f, D}\left(\tau_{4}\right)+E_{f, B}\left(\tau_{5}\right)-E_{f, B}\left(\tau_{6}\right)+\Delta t P_{f, \sigma}$

subejct to the constraints above. The quantity $P_{f, \sigma}$ is the fuel power for the engine operating point corresponding to a constant vehicle speed $v_{\sigma}$.

To summarize, the ED-OCP is reduced to a one-dimensional parametric optimization for the complete four-mode sequence. For speed profiles of three-mode basic sequences, there is even no optimization necessary at all. In this case, the velocity trajectory is given by the mission constraints only. 
The online algorithm used in the online assessment system presented in this article operates as follows; see Figure S7. In a first step, the vehicle parameters are set, including information input by the user concerning the vehicle weight, the type of engine, its rated power, and swept volume. Other data, such as the fuel map, are extrapolated from typical engines by scaling with respect to the size. Best-efficiency torque curves are calculated from the fuel map. From such information, the speed, position, and fuel trajectories for the three modes A, D, and B are preliminarly calculated as described above.

During the online use, the identification of the boundary conditions of the actual segment $\left(v_{i}, v_{f}, t_{f}, s_{f}\right)$ allow a reduction of the number of mode sequences admissible. When several sequences are still possible, the rest of the algorithm is repeated and the results are compared to find the optimal mode sequence. For three-mode sequences, constraints are directly enforced and all switching times are calculated. For fourmode sequences, the degree of freedom, chosen as to be the switching time $t_{3}$ (or $\tau_{5}$ ), is found by finding the minimum of the function (57). The search limits are $t_{3, \min }$ such that $v_{B}\left(\tau_{5, \min }\right)=v_{\sigma}$ and $t_{3, \max }=t_{f}$. Within these boundaries, the function $E_{f}\left(t_{3}\right)$ is a unimodal in most practical situations and thus the internal halving algorithm, a direct search method, has been selected to find its minimum.

\section{Author Information}

Antonio Sciarretta (antonio.sciarretta@ifpen.fr) received a M.Sc. (Mech. Eng.) degree in 1995, and a Doctorate degree (Thermal machines) in 1999, both from the University of L'Aquila, Italy. He earned a Habilitation (Control Eng.) in 2009 from the Grenoble Institute of Technology, France. After several teaching and research experiences in Italy and Switzerland, particularly at ETH Zurich of which he is still a scientific collaborator, he joined the Control, Signals and Systems Department of IFP Energies Nouvelles in 2006. Since 2009 he is IFPEN Expert for "Hybrid vehicles and energy management" as well as holder of the Tuck foundation Chair on the same subject at IFP School. He has actively contributed to the development of hybrid-vehicle activities at IFPEN and he is currently managing research activities on control and optimization for electrified vehicles and other energy systems. He has coauthored the Springer book "Vehicle Propulsion Systems" (three edi- 
tions) and contributes to the scientific and professional community as a journal associate editor, conference organizer, and project evaluator.

Giovanni De Nunzio received a B.Sc. and a M.Sc. (Information and Automation Engineering) in 2007 and 2010 respectively, at the University of L'Aquila, Italy. He completed a research internship at Magneti Marelli Powertrain, Italy, on the estimation of $\mathrm{NO}_{x}$ emissions in 2010, and a research assistantship at West Virginia University, USA, on the control of UAVs in 2011. He is currently a Ph.D. student at IFP Energies Nouvelles and INPG, France. His research activities focus on the control of intelligent vehicles in urban traffic and the energy consumption minimization via eco-driving algorithms.

Luis Leon Ojeda received the B.Sc. degree in Electrical Engineering from the Universidad Central de Venezuela, in 2006. He received his M.S. degree in Systems Engineering from the University La Sapienza, Roma, Italy, in 2008, and the Ph.D. in Automatic Control from the University of Grenoble, France, in July 2014. He is currently a research engineer at the IFP Energies Nouvelles. His research interests are in the area of intelligent transportation systems, specifically, traffic estimation, forecasting, and data treatment. 
Figures

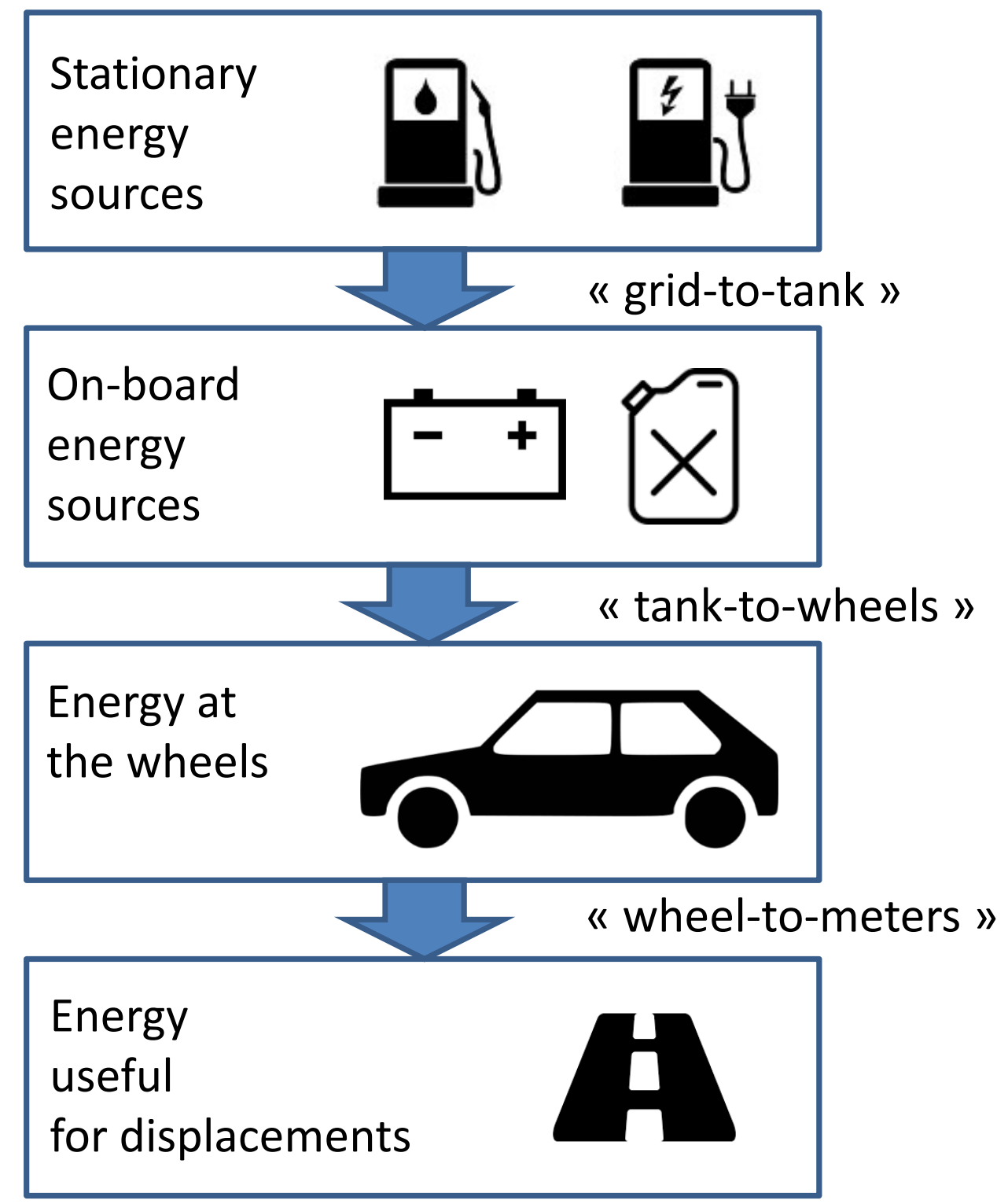

Figure 1: The three main energy conversion steps for road vehicles. 

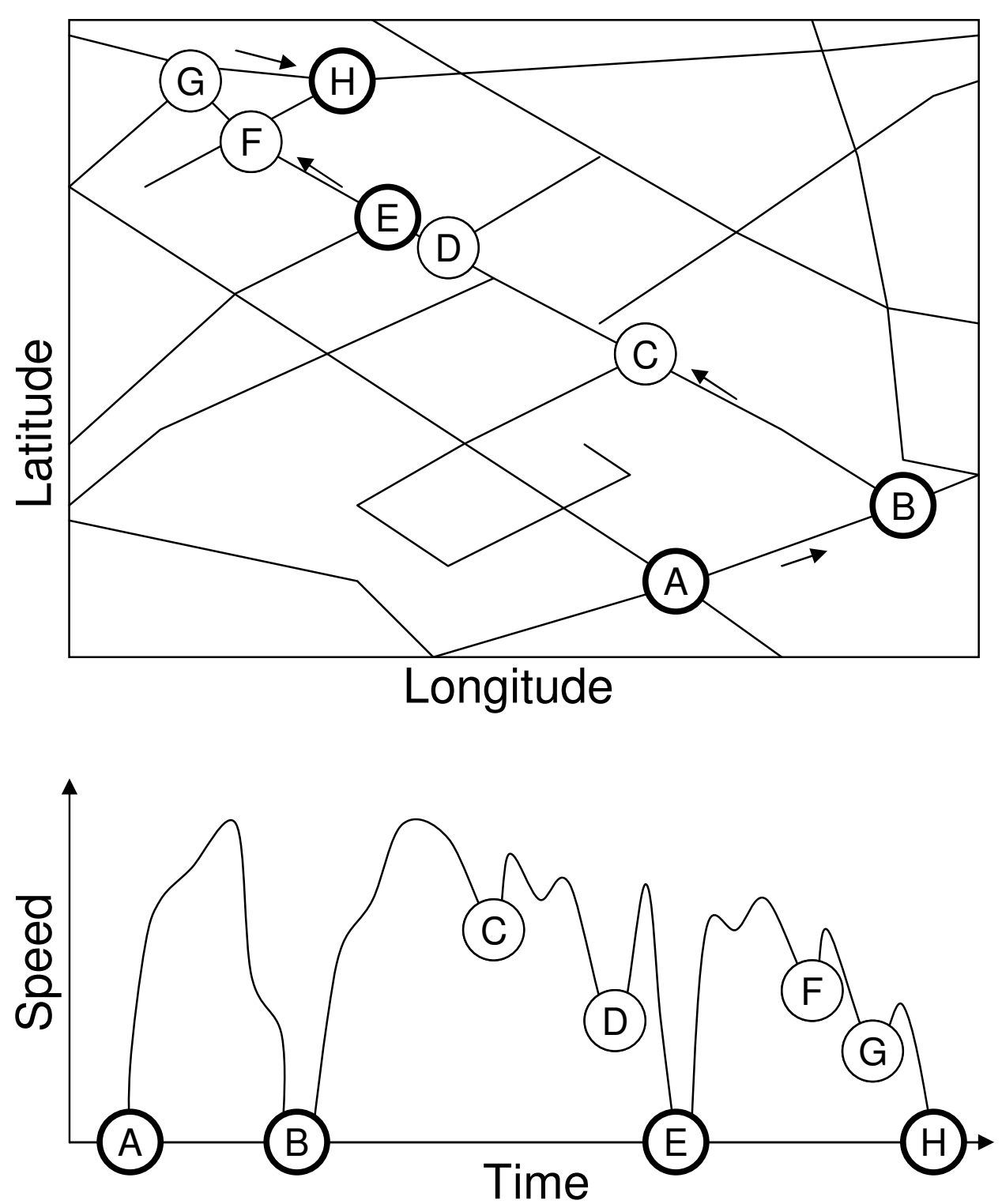

Figure 2: An example urban trip (top) and corresponding vehicle speed (bottom) with indication of the breakpoints A-H (A:start; B:intersection without priority; C,D,F,G: intersections with priority; E:traffic light; H:stop) and seven segments (A-B, B-C, etc.). From [28]. 


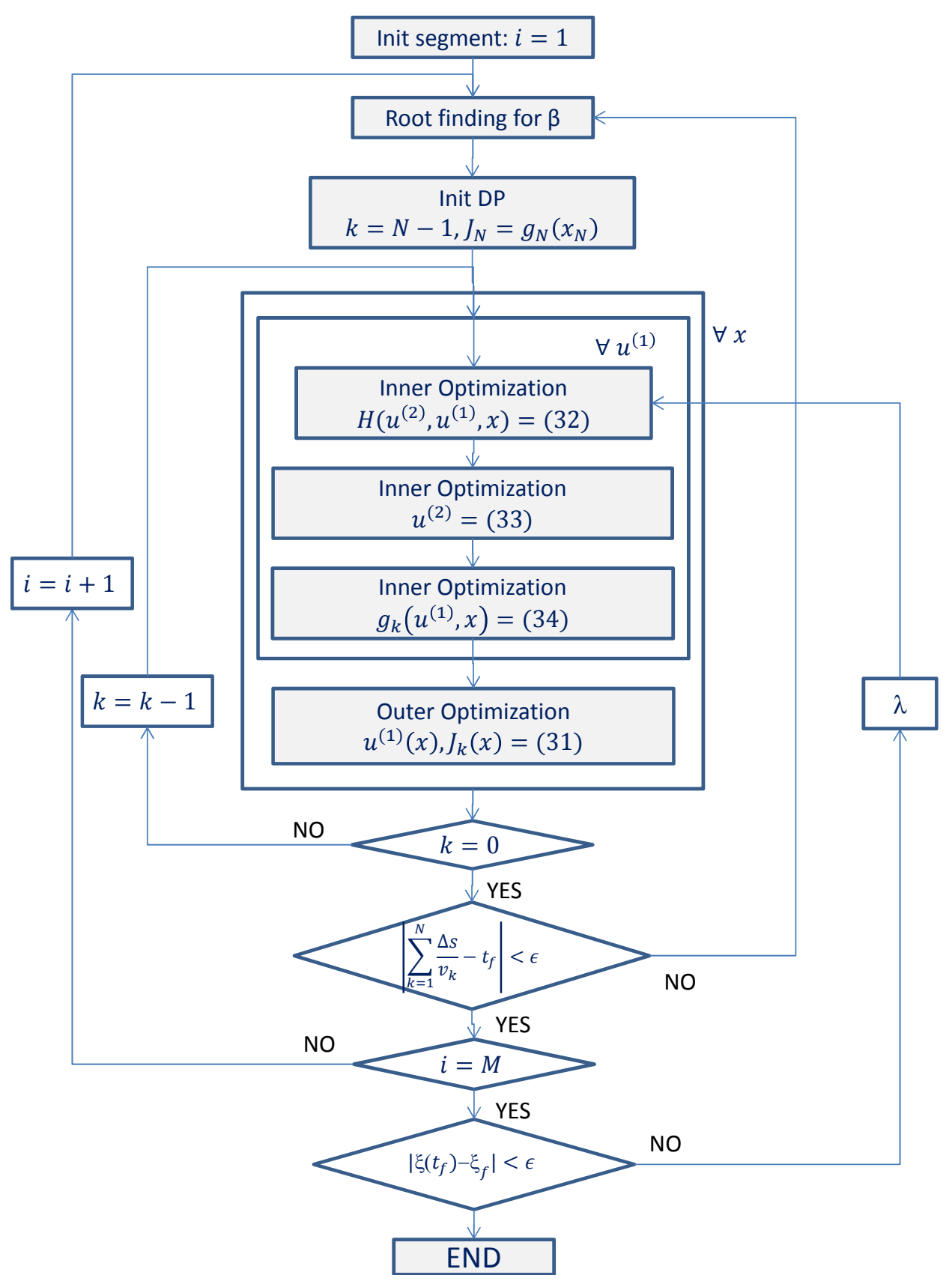

Figure 3: Flowchart of a bi-level optimization algorithm for HEVs. Numbers in brackets refer to equations in this article. 


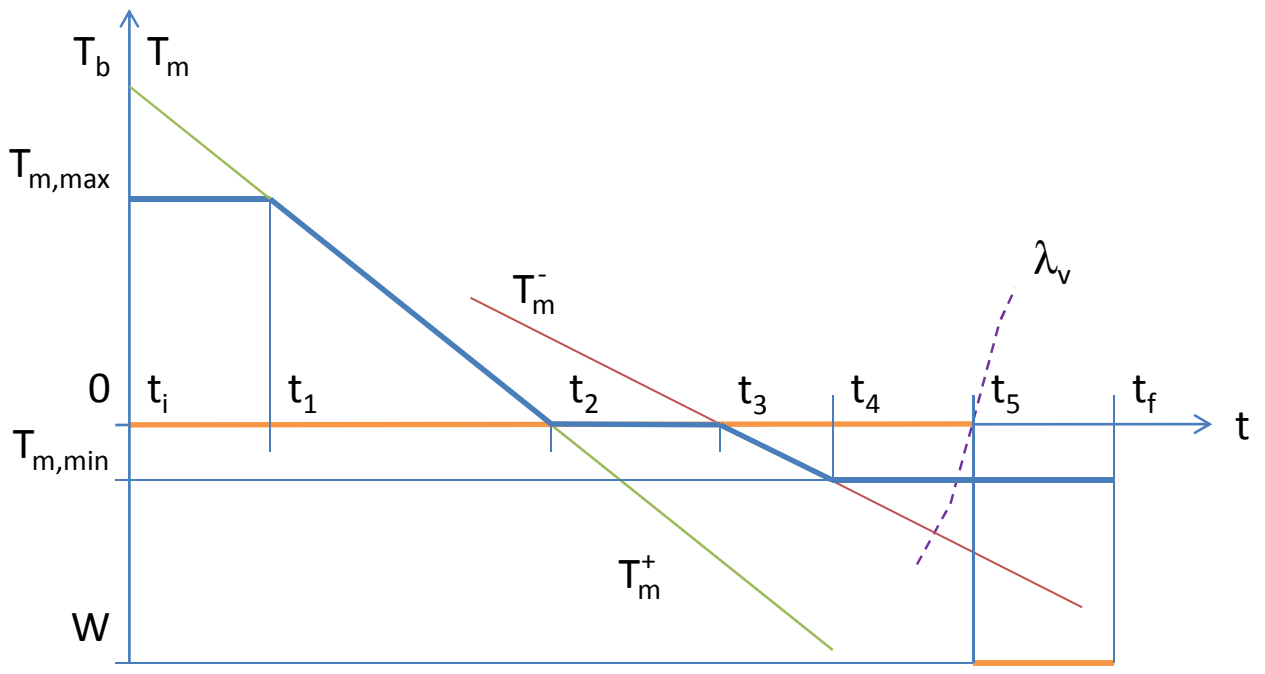

Figure 4: Qualitative control trajectories for an EV, for the mode sequence SA-A-C-D-SD-B. Shown in the picture are motor and brake torques $T_{m}^{*}$ (blue), $T_{b}^{*}$ (orange), auxiliary functions $T_{m}^{+}$(green) and $T_{m}^{-}$(brown) defined in the main text, and velocity costate $\lambda_{v}$ (dashed purple) as a function of time. Switching times are such that $T_{m}^{+}\left(t_{1}\right)=T_{m, \max }, T_{m}^{+}\left(t_{2}\right)=0, T_{m}^{-}\left(t_{3}\right)=$ $0, T_{m}^{-}\left(t_{4}\right)=T_{m, \min }, \lambda_{v}\left(t_{5}\right)=0$. 


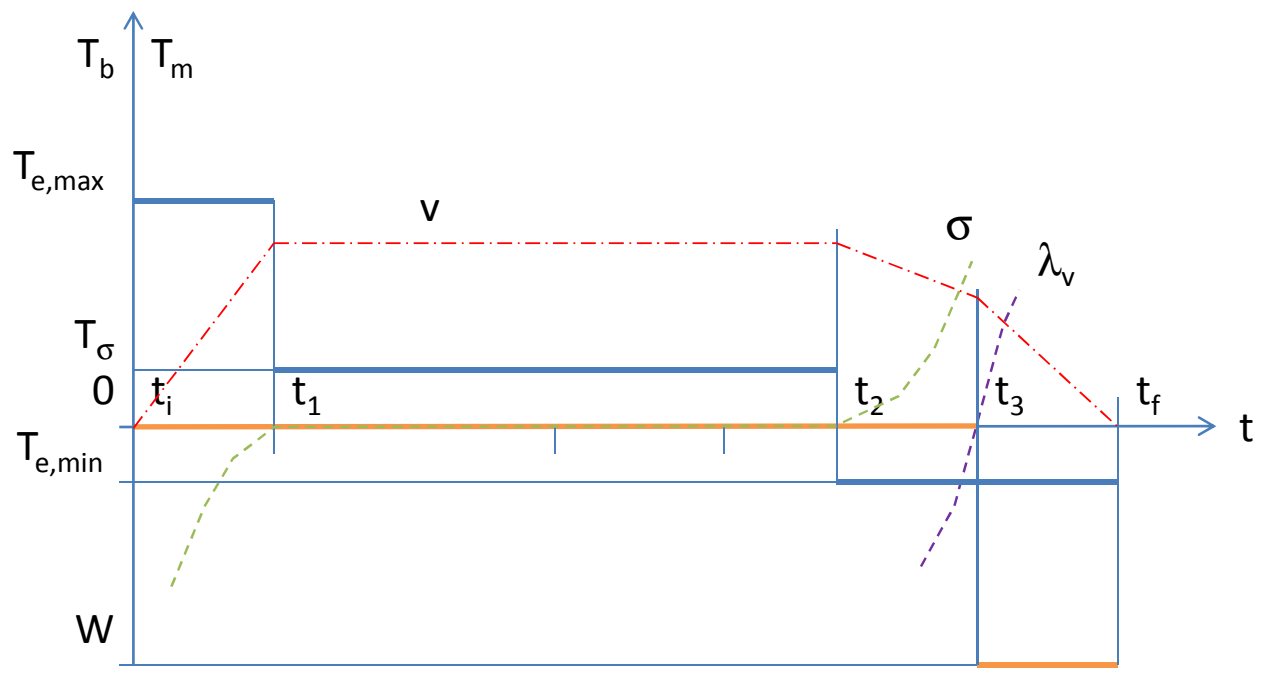

Figure 5: Qualitative control and speed trajectories for an ICEV, for the mode sequence A-C-D-B. Shown in the picture are engine and brake torques, $T_{e}$ (blue), $T_{b}$ (orange), vehicle speed $v$ (dashdot red), auxiliary function $\sigma$ (dashed green) defined in the main text, and velocity costate $\lambda_{v}$ (dashed purple) as a function of time. Switching times are such that $\sigma\left(t_{1}\right)=0$, $\sigma\left(t_{2}\right)=0, \lambda_{v}\left(t_{3}\right)=0$. 
Table 1: Relative influence of vehicle parameters and driving profiles on the wheel-to-meters energy efficiency of a typical full-size passenger car. Sensitivity is defined as the percent reduction of energy consumption per percent reduction of the considered variable. The calculation method is detailed and illustrated in [1].

\begin{tabular}{ll}
\hline Vehicle parameter & Sensitivity $[-]$ \\
\hline Weight & 0.7 \\
Rolling friction & 0.35 \\
Aerodynamics & 0.3 \\
\hline Driving profile parameter & Sensitivity $[-]$ \\
\hline Mean square speed & 0.6 \\
Mean acceleration & 0.35 \\
\hline
\end{tabular}



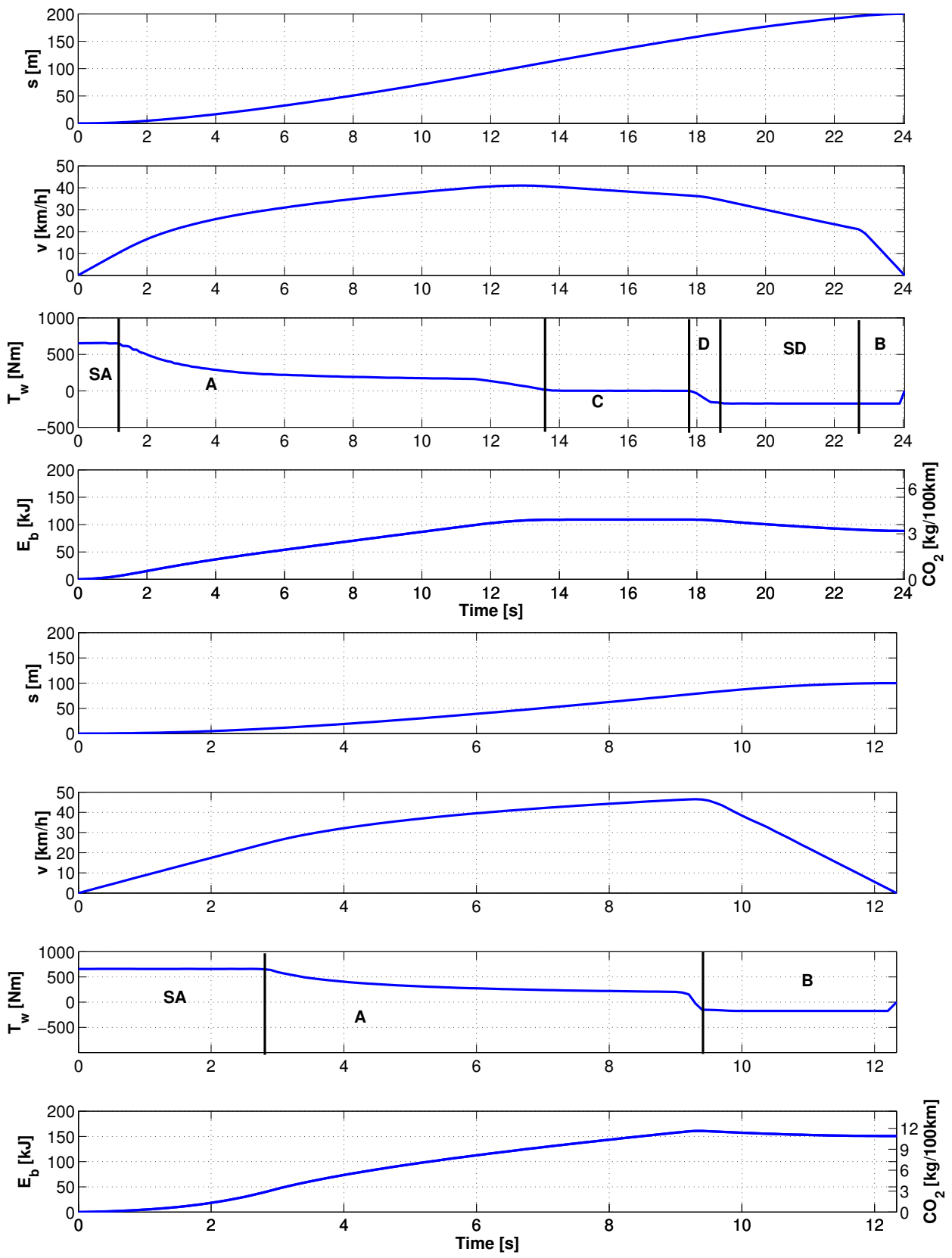

Figure 6: DP trajectories for an EV, $s_{f}=200 \mathrm{~m}, t_{f}=24 \mathrm{~s}$ (top), $s_{f}=100$ $\mathrm{m}, t_{f} \approx 12 \mathrm{~s}$ (bottom), $v_{i}=v_{f}=\alpha=0$. From top to bottom, the figures show position $s$, speed $v$, torque at the wheels $T_{w}:=r_{w} F_{w}$, and battery energy consumption $E_{b}$ as a function of time. Labels to the wheel torque refer to the control modes introduced in the main text. 

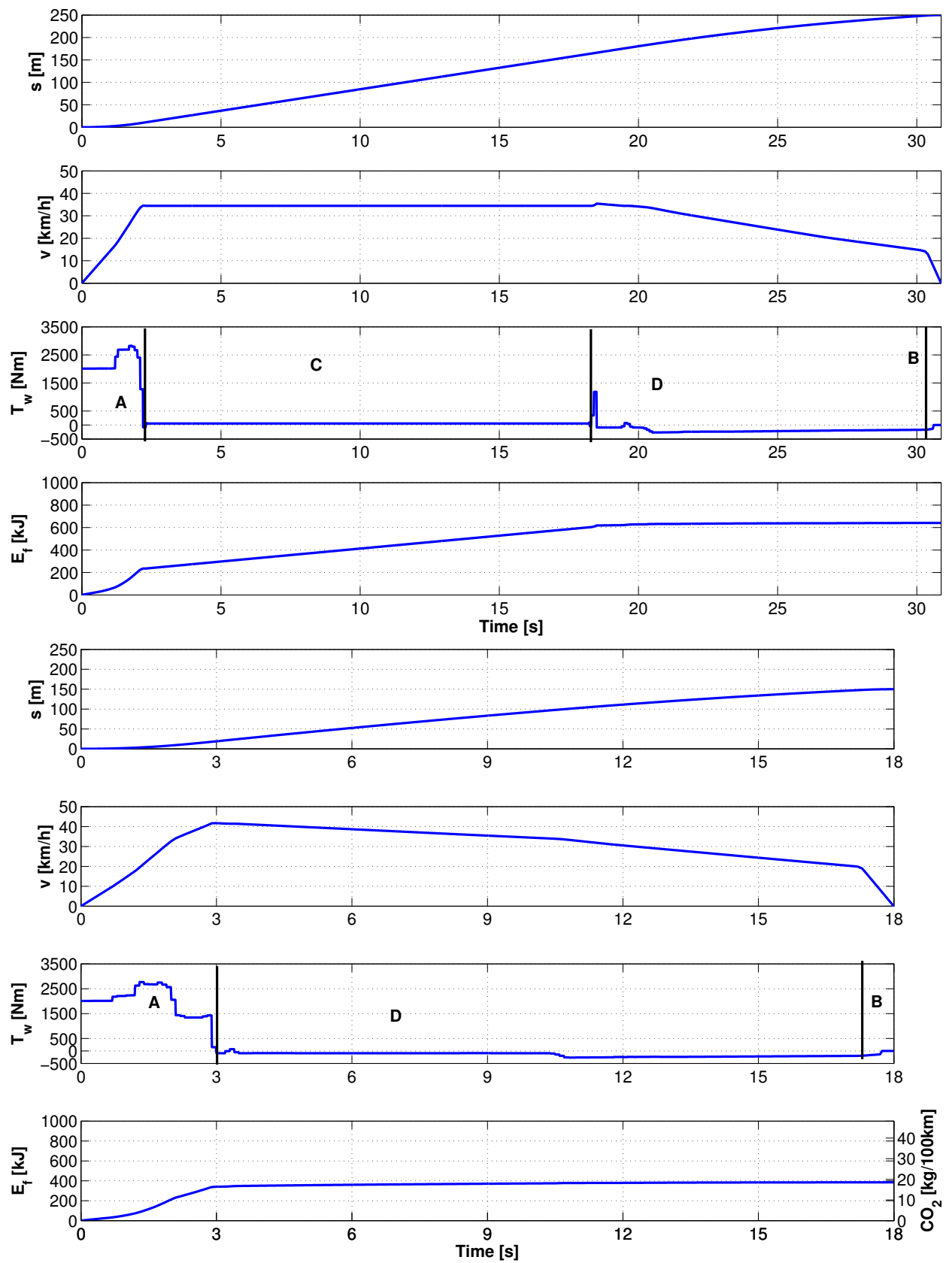

Figure 7: DP trajectories for an ICEV $, s_{f}=250 \mathrm{~m}, t_{f} \approx 30 \mathrm{~s} \mathrm{(top),} s_{f}=150$ $\mathrm{m}, t_{f}=18 \mathrm{~s}$ (bottom), $v_{i}=v_{f}=\alpha=0$. From top to bottom, the figures show position $s$, speed $v$, torque at the wheels $T_{w}:=r_{w} F_{w}$, and fuel energy consumption $E_{f}$ as a function of time. Labels to the wheel torque refer to the control modes introduced in the main text. 

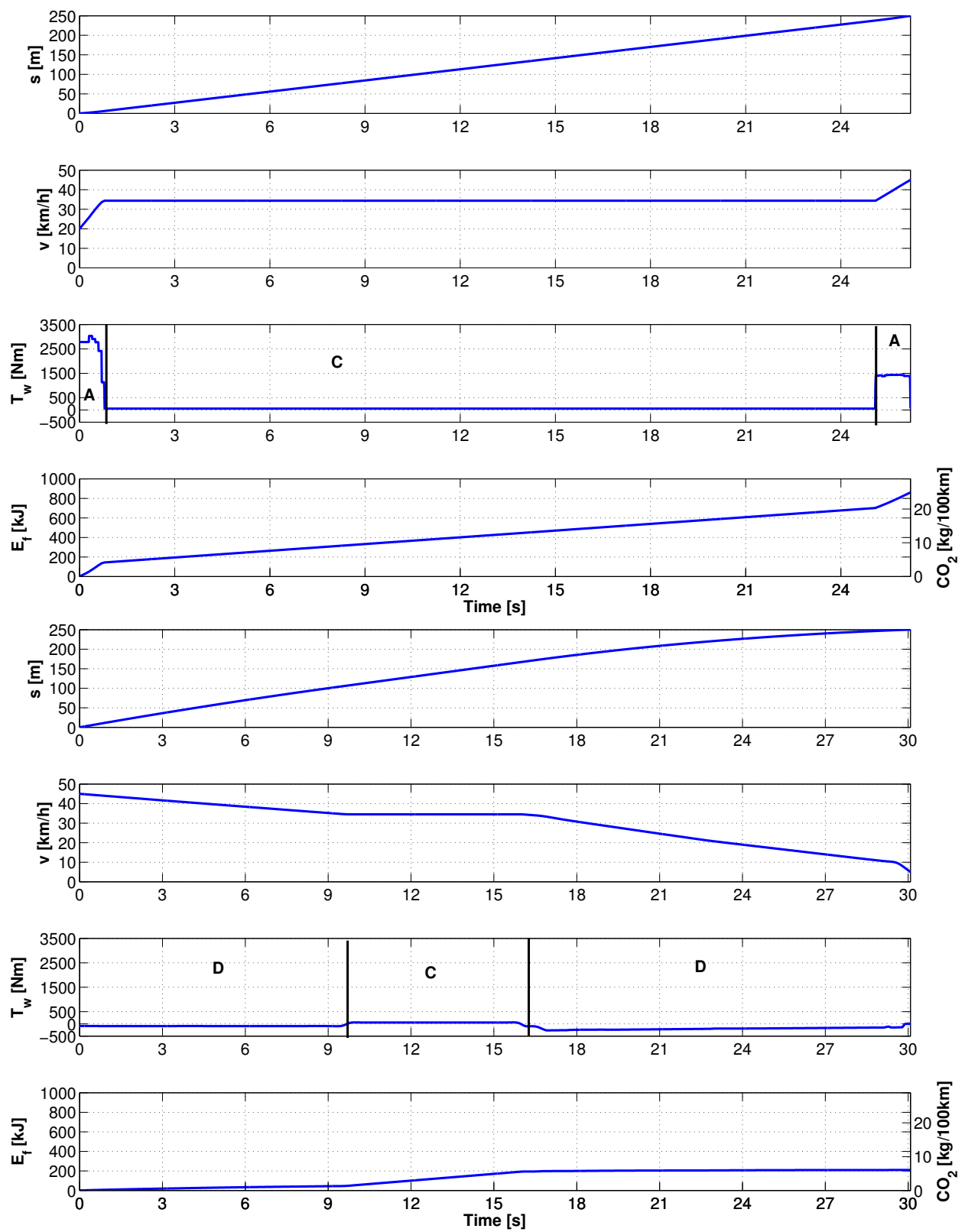

Figure 8: DP trajectories for an ICEV, $v_{i}=20 \mathrm{~km} / \mathrm{h}, v_{f}=45 \mathrm{~km} / \mathrm{h}$, $t_{f} \approx 26 \mathrm{~s}$ (top), $v_{i}=45 \mathrm{~km} / \mathrm{h}, v_{f}=5 \mathrm{~km} / \mathrm{h}, t_{f}=30 \mathrm{~s}$ (bottom), $s_{f}=250$ $\mathrm{m}, \alpha=0$. From top to bottom, the figures show position $s$, speed $v$, torque at the wheels $T_{w}:=r_{w} F_{w}$, and fuel energy consumption $E_{f}$ as a function of time. Labels to the wheel torque refer to the control modes introduced in the main text. 


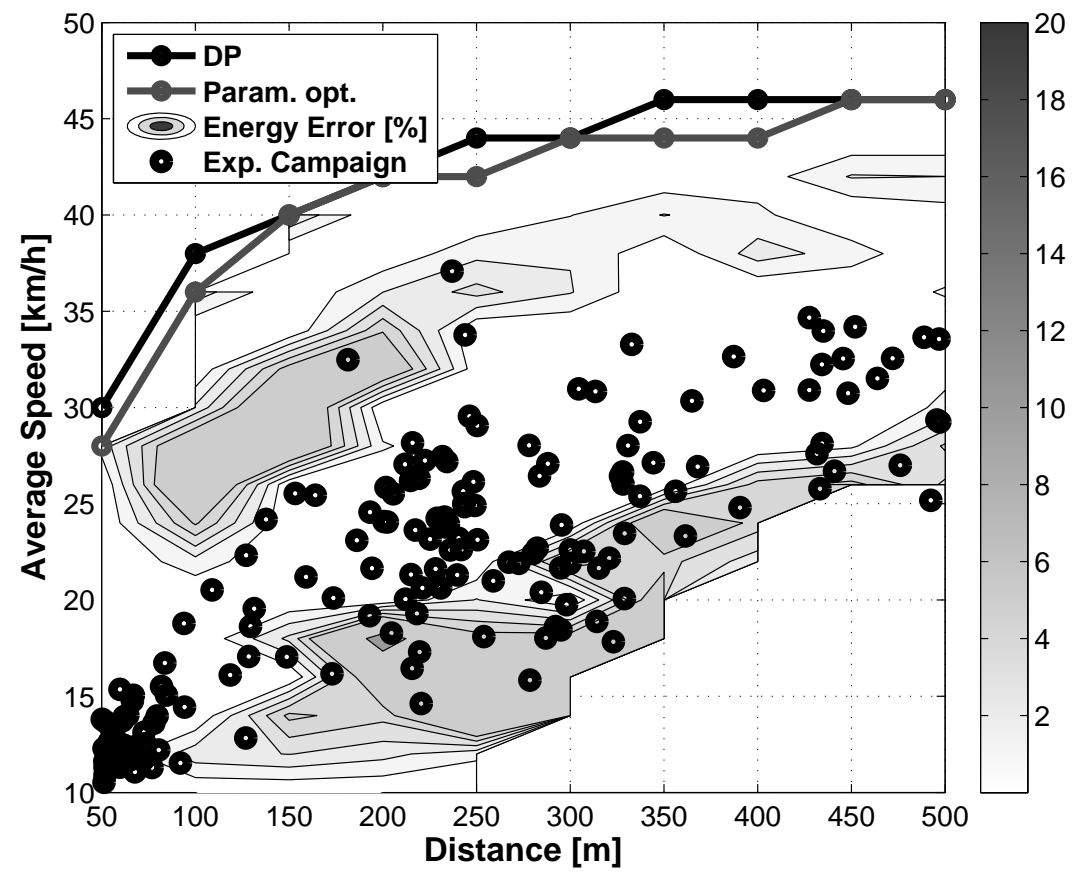

Figure 9: Differences between fuel energy consumption of an ICEV as calculated with the DP and with the parametric optimization presented in "Parametric Optimization Method for ICEVs". Also shown are operating conditions (in terms of distance and average speed) of a typical experimental campaign, see Figure 11. 

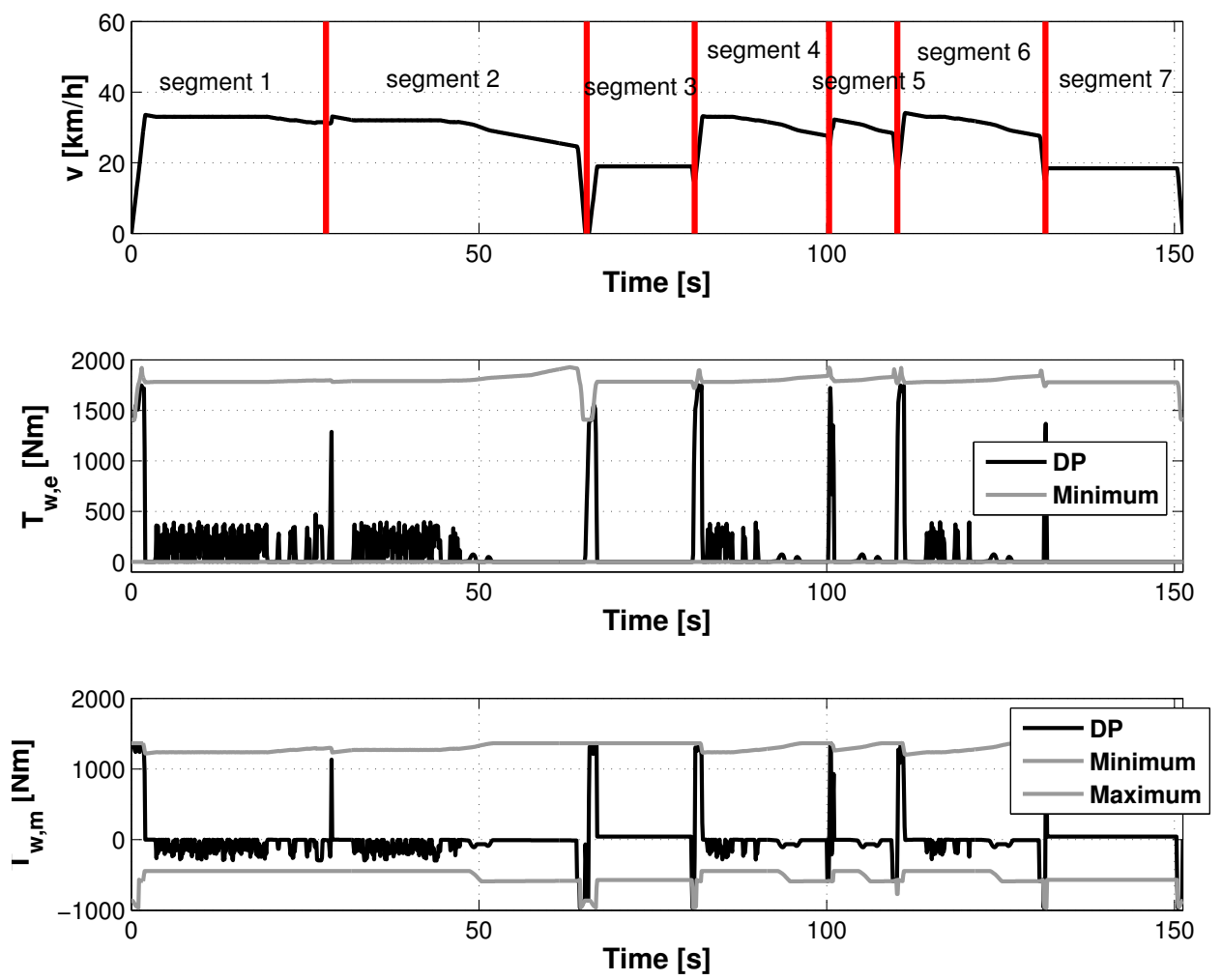

Figure 10: DP trajectories for an HEV, seven consecutive segments. From top to bottom, the figures show speed $v$, torque at the wheels from the engine, $T_{w, e}:=T_{e} \gamma_{e}$, and torque at the wheels from the motor $T_{w, m}:=T_{m} \gamma_{m}$ as a function of time. 


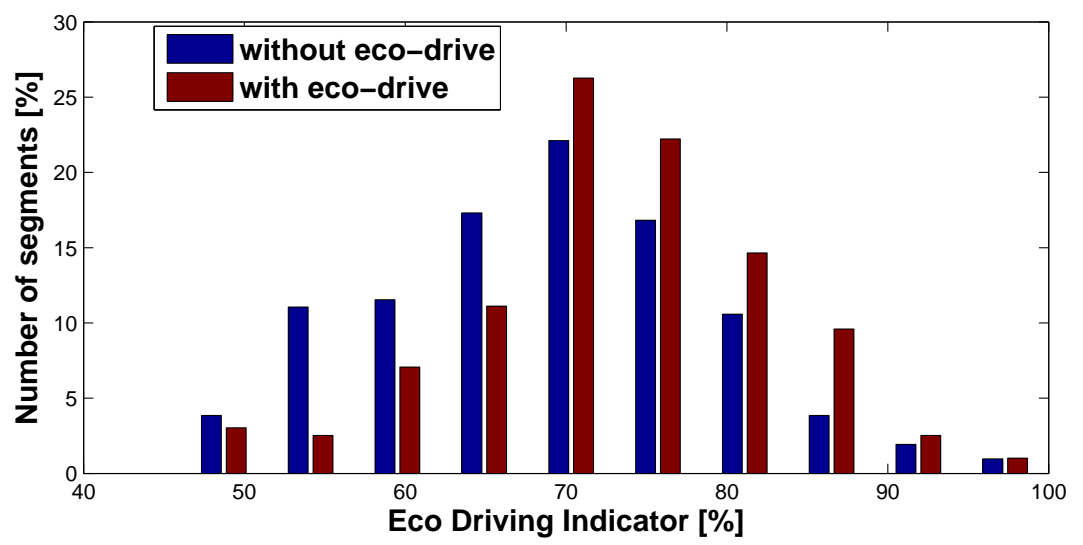

Figure 11: Experimental results obtained with optimal ED online assessment, for an EV: percentage of number of segments as a function of the indicator defined as $\frac{E_{b}^{*}}{E_{b}}$ [28].
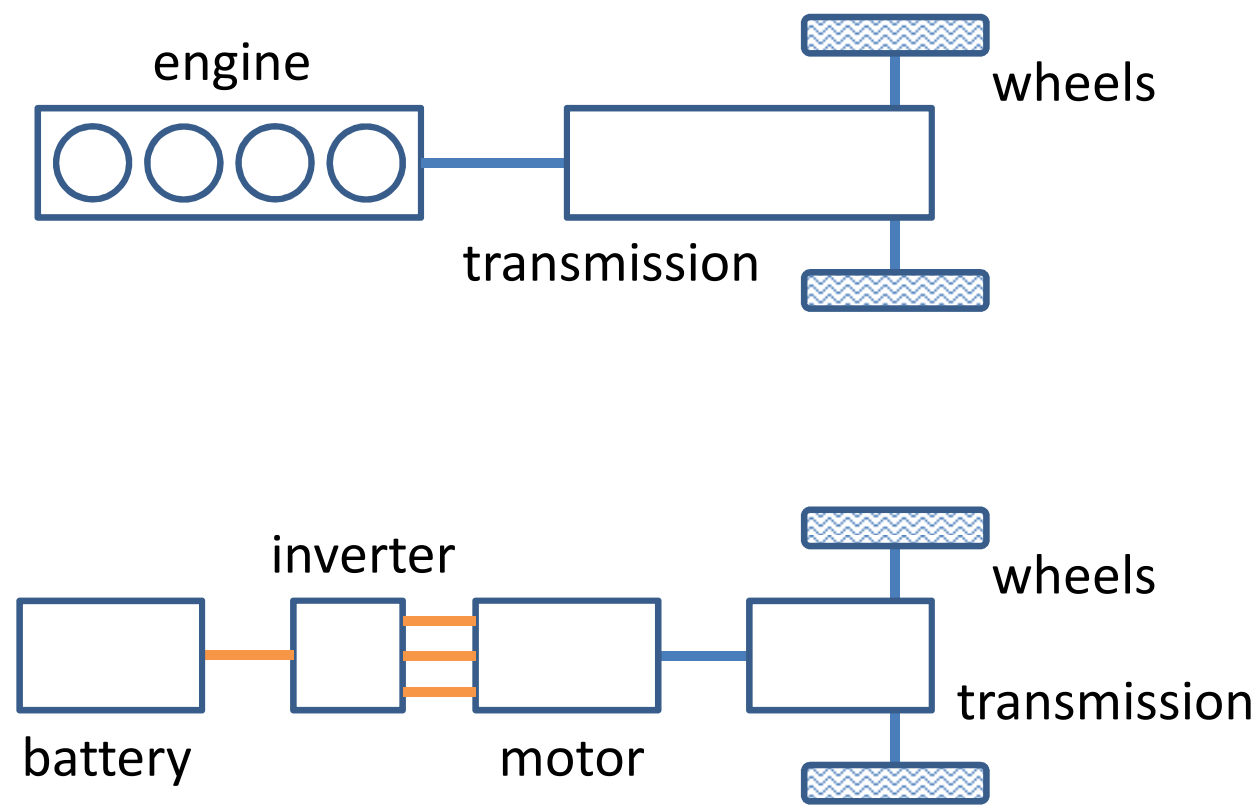

Figure S1: General layout of an ICEV (top) and an EV (bottom). Blue bold lines between subsystems represent mechanical power, orange bold lines represent electrical power. 


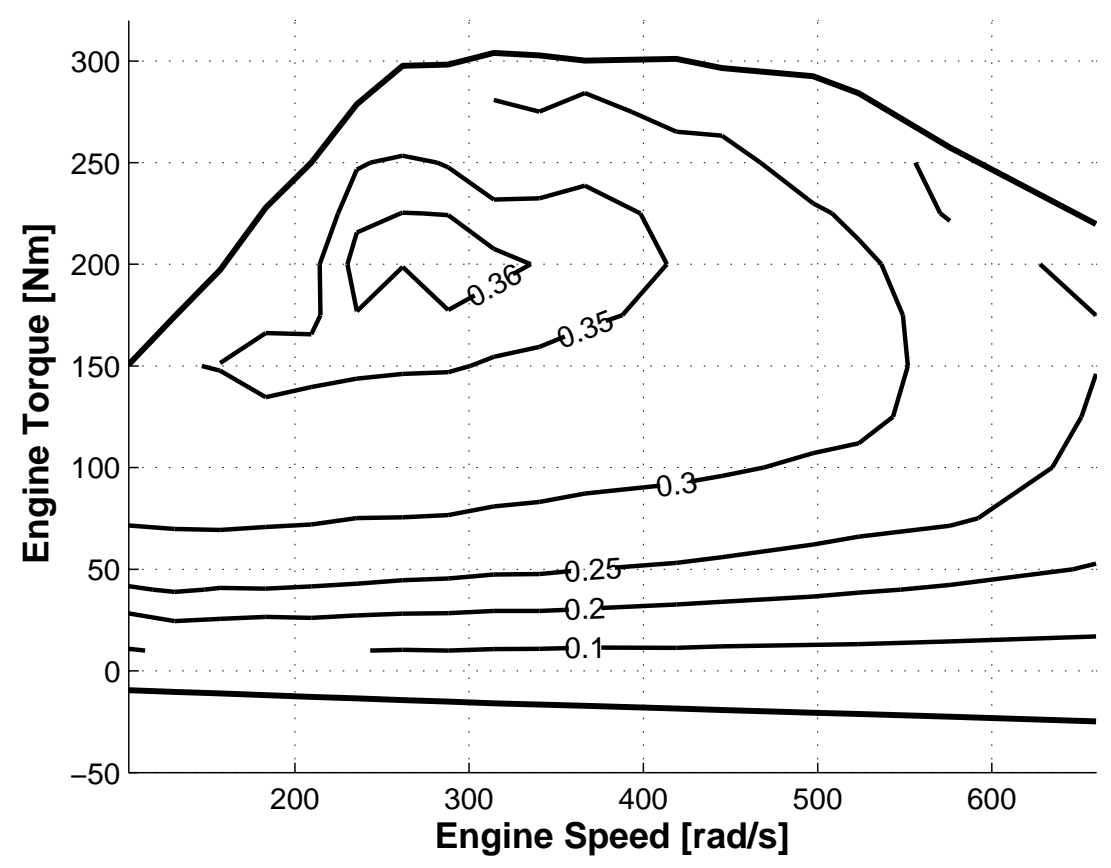

Figure S2: Typical efficiency map of an ICEV engine. The figure shows equal efficiency contours (labelled), as well as maximal torque curve $T_{e, \max }$ and minimal torque curve $T_{e, \min }$. 


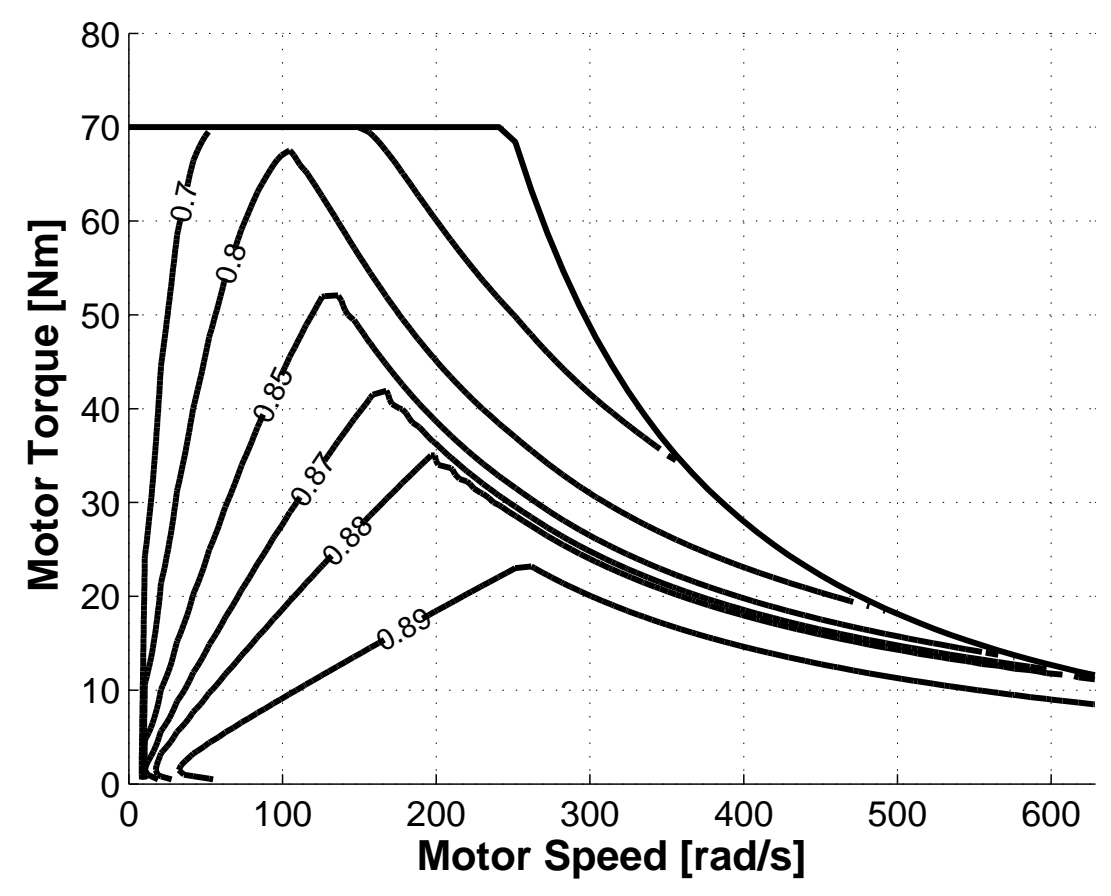

Figure S3: Typical efficiency map of an EV electric motor. The figure shows equal efficiency contours (labelled), as well as maximal torque curve $T_{m, \max }$. When specific data are not available, second quadrant, that is, negative torque values (generating mode) may be estimated by mirroring the first quadrant. 

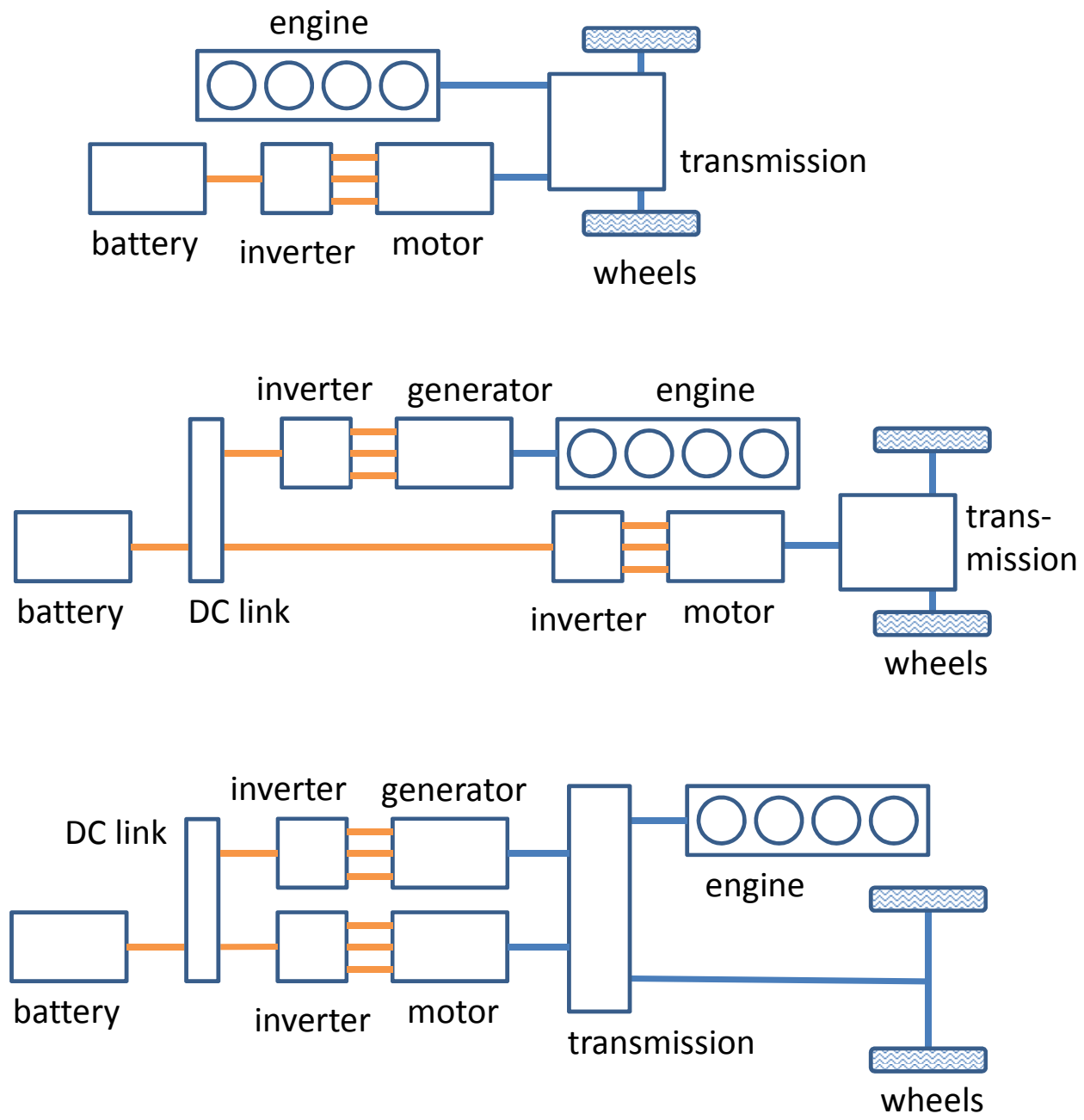

Figure S4: General layout of a parallel HEV (top), a series HEV (middle), and a series-parallel HEV (bottom). Blue bold lines between subsystems represent mechanical power, orange bold lines represent electrical power. 

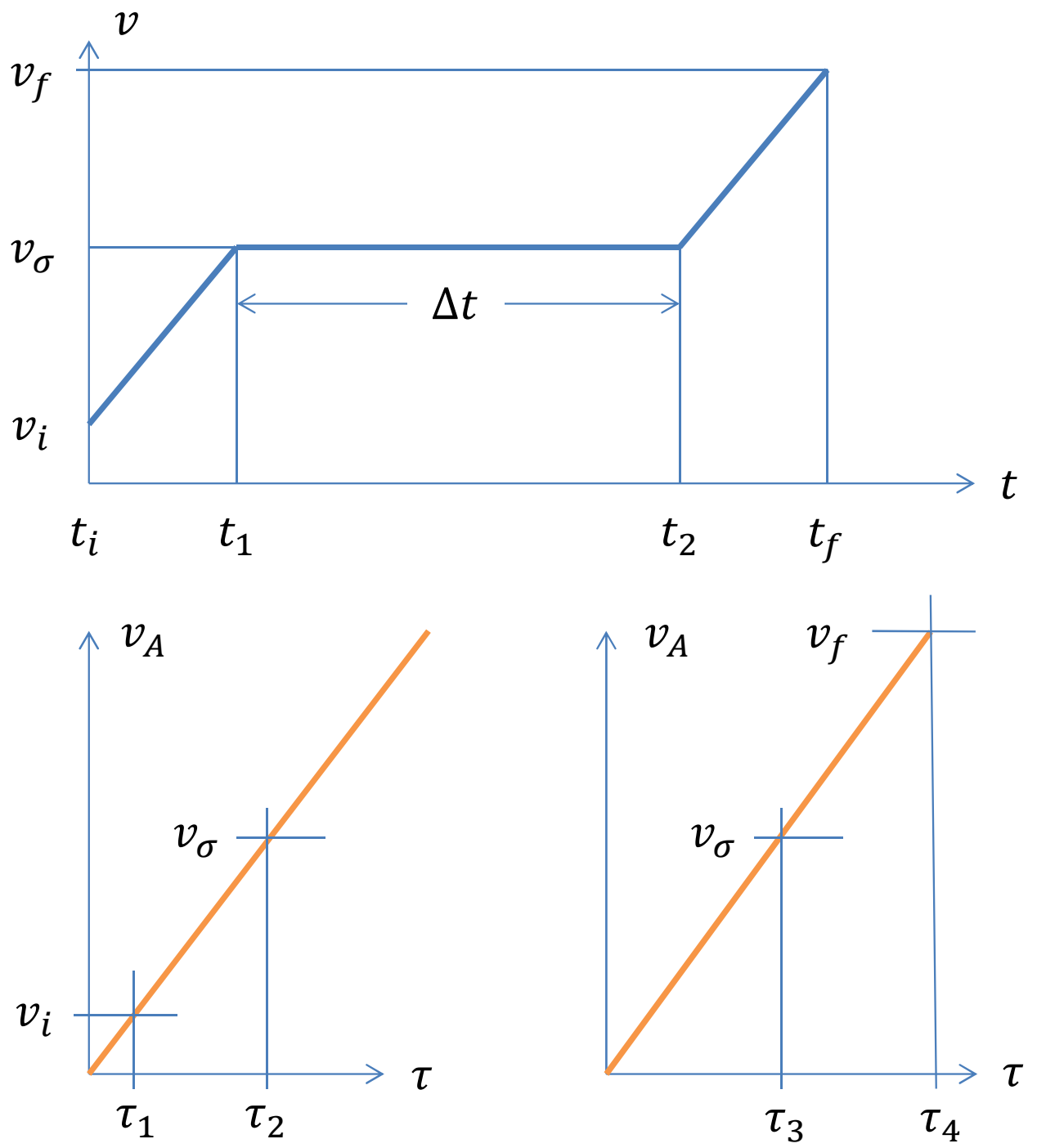

Figure S5: Graphical illustration of the parametric optimization method in the case of a three-phase optimal trajectory. Upper plot shows the speed trajectory with the switching times $t_{1}, t_{2}$ and the singular arc $v_{\sigma}$. Lower plots show the trajectory $v_{A}(\tau)$ together with the characteristic times $\tau_{1}, \ldots, \tau_{4}$. 

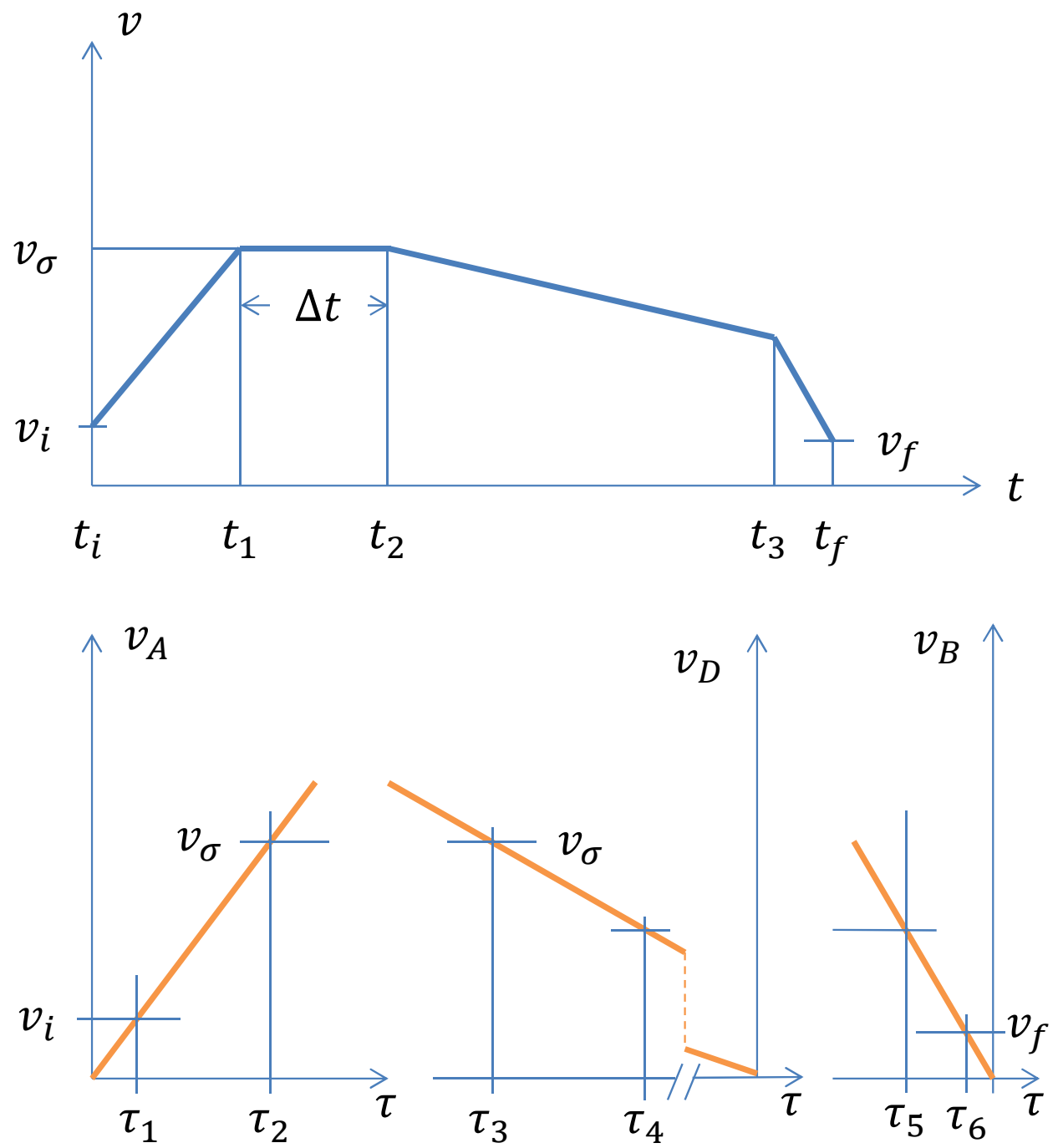

Figure S6: Graphical illustration of the parametric optimization method in the case of a four-phase optimal trajectory. Upper plot shows the speed trajectory with the switching times $t_{1}, \ldots, t_{3}$ and the singular arc $v_{\sigma}$. Lower plots show the trajectories $v_{A}(\tau), v_{D}(\tau)$, and $v_{B}(\tau)$ together with the characteristic times $\tau_{1}, \ldots, \tau_{6}$. 


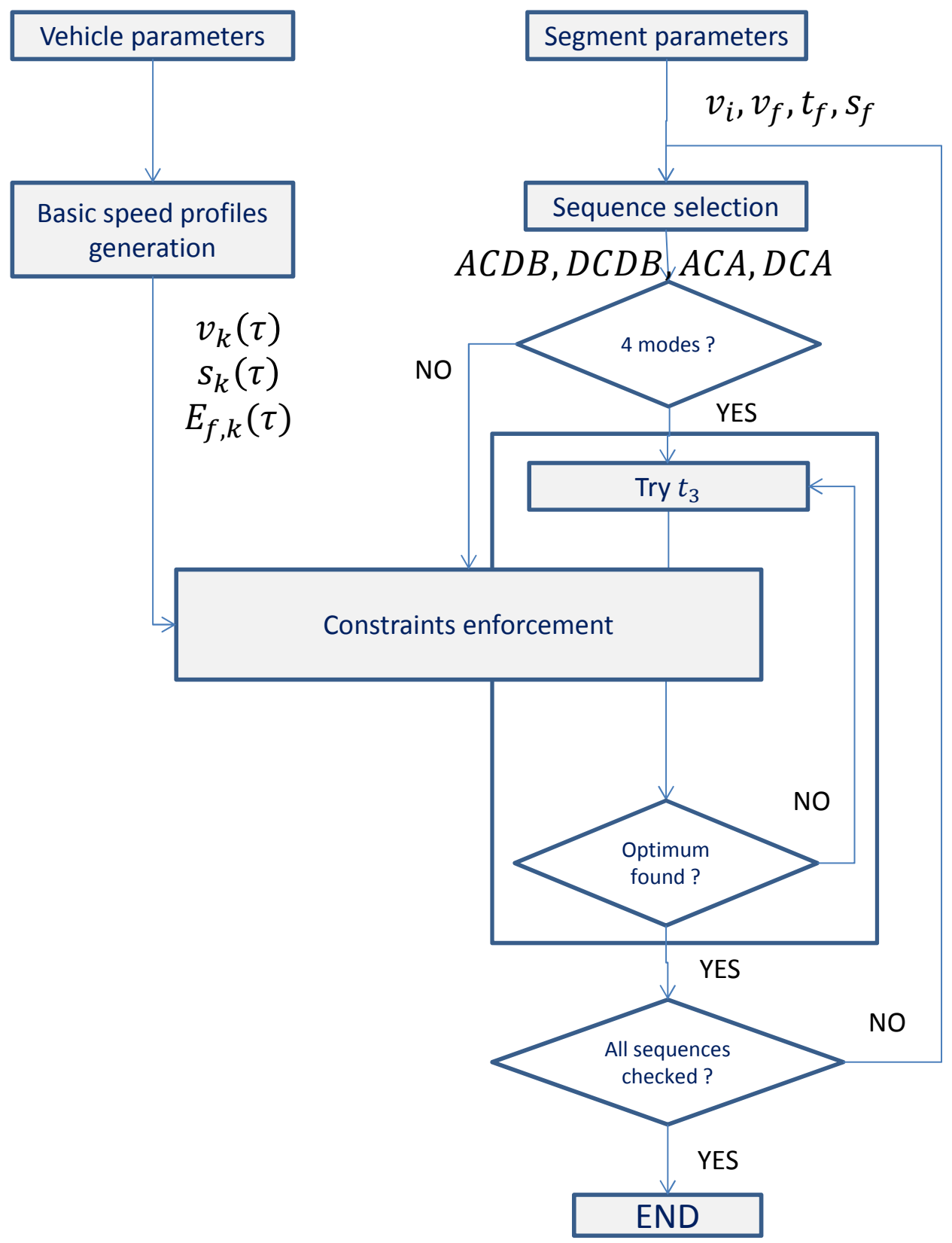

Figure S7: Flowchart of the parametric optimization algorithm for ICEVs. 